OPEN ACCESS

Edited by:

Michael Lazarus,

University of Tsukuba, Japan

Reviewed by:

Stewart Alan Anderson, University of Pennsylvania,

United States

Gavin John Clowry,

Newcastle University,

United Kingdom

*Correspondence:

Fu-Chin Liu

fuchin@ym.edu.tw

${ }^{\dagger}$ These authors have contributed equally to this work

Received: 19 February 2021 Accepted: 12 April 2021

Published: 13 May 2021

Citation:

Chang $C-C$, Kuo H-Y, Chen S-Y, Lin W-T, Lu K-M, Saito T and Liu F-C

(2021) Developmental

Characterization of

Schizophrenia-Associated Gene

Zswim6 in Mouse Forebrain.

Front. Neuroanat. 15:669631. doi: 10.3389/fnana.2021.669631

\section{Developmental Characterization of Schizophrenia-Associated Gene Zswim6 in Mouse Forebrain}

\author{
Chuan-Chie Chang ${ }^{1 \dagger}$, Hsiao-Ying Kuo ${ }^{2 \dagger}$, Shih-Yun Chen ${ }^{1 \dagger}$, Wan-Ting Lin ${ }^{1}$, \\ Kuan-Ming Lu ${ }^{1}$, Tetsuichiro Saito ${ }^{3}$ and Fu-Chin Liu ${ }^{1 *}$
}

${ }^{1}$ Institute of Neuroscience, National Yang Ming Chiao Tung University, Taipei, Taiwan, ${ }^{2}$ Department of Biological Science and Technology, National Yang Ming Chiao Tung University, Taipei, Taiwan, ${ }^{3}$ Department of Developmental Biology, Graduate School of Medicine, Chiba University, Chiba, Japan

Schizophrenia is a devastating neuropsychiatric disease with a globally $1 \%$ life-long prevalence. Clinical studies have linked Zswim6 mutations to developmental and neurological diseases, including schizophrenia. Zswim6's function remains largely unknown. Given the involvement of Zswim6 in schizophrenia and schizophrenia as a neurodevelopmental disease, it is important to understand the spatiotemporal expression pattern of Zswim6 in the developing brain. Here, we performed a comprehensive analysis of the spatiotemporal expression pattern of Zswim6 in the mouse forebrain by in situ hybridization with radioactive and non-radioactivelabeled riboprobes. Zswim6 mRNA was detected as early as E11.5 in the ventral forebrain. At E11.5-E13.5, Zswim6 was highly expressed in the lateral ganglionic eminence (LGE). The LGE consisted of two progenitor populations. Dlx ${ }^{+}$;Er81 ${ }^{+}$cells in dorsal LGE comprised progenitors of olfactory bulb interneurons, whereas $\mathrm{Dlx}^{+} ; \mathrm{|S} \mid 1^{+}$ progenitors in ventral LGE gave rise to striatal projection neurons. Zswim6 was not colocalized with Er81 in the dorsal LGE. In the ventral LGE, Zswim6 was colocalized with striatal progenitor marker Nolz-1. Zswim6 was highly expressed in the subventricular zone (SVZ) of LGE in which progenitors undergo the transition from proliferation to differentiation. Double labeling showed that Zswim6 was not colocalized with proliferation marker Ki67 but was colocalized with differentiation marker Tuj1 in the SVZ, suggesting Zswim6 expression in early differentiating neurons. Zswim6 was also expressed in the adjacent structures of medial and caudal ganglionic eminences (MGE, CGE) that contained progenitors of cortical interneurons. At E15.5 and E17.5, Zswim6 was expressed in several key brain regions that were involved in the pathogenesis of schizophrenia, including the striatum, cerebral cortex, hippocampus, and medial habenular nucleus. Zswim6 was persistently expressed in the postnatal brain. Cell type analysis indicated that Zswim6 mRNA was colocalized with $D 1 R$-expressing striatonigral and D2R-expressing striatopallidal neurons of the adult striatum with a higher colocalization in striatopallidal neurons. 
These findings are of particular interest as striatal dopamine D2 receptors are known to be involved in the pathophysiology of schizophrenia. In summary, the comprehensive analysis provides an anatomical framework for the study of Zswim6 function and Zswim6-associated neurological disorders.

Keywords: basal ganglia, striatum, schizophrenia, dopamine, Zswim

\section{INTRODUCTION}

The members of ZSWIM family contain a SWIM domain of $\mathrm{CxCxnCxH}$ motif of predicted zinc chelating residues that may be involved in DNA binding and protein-protein interaction (Makarova et al., 2002). ZSWIM gene family consists of 10 members, MAP3K1 and ZSWIM1 to ZSWIM9 (Makarova et al., 2002). Of the Zswim family members, Zswim6 is the member that is expressed at a high level in the adult mouse brain (Lein et al., 2007). A previous study of Zswim6 knockout mice indicates that Zswim6 regulates the morphology of striatal neurons and motor function (Tischfield et al., 2017). In addition to the striatum, Zswim6 is also expressed in the amygdala. NMDA-induced downregulation of Zswim6 was observed in the central nucleus of amygdala of Fyn knockout mice (Kai et al., 2006).

The biological importance of Zswim6 has been implicated in studies of diseases. Clinical studies have shown that patients with Zswim6 genetic mutations are associated with developmental and neurological diseases, including schizophrenia, acromelic frontonasal dysostosis and intellectual disability (Ripke et al., 2013; Lencz et al., 2014; Schizophrenia Working Group of the Psychiatric Genomics Consortium, 2014; Smith et al., 2014; Twigg et al., 2016; Palmer et al., 2017). Interestingly, ZSWIM6 is ranked in the top five schizophrenia-associated genes that are linked to the MAPK signaling pathway (Pers et al., 2016). An animal model study has shown that Zswim6 is downregulated in the amygdala of MECP2 duplicated mice, a mouse model of MECP2 duplication syndrome (Samaco et al., 2012).

We have previously characterized the expression pattern of Zswim5, a paralogue of Zswim6, in the developing mouse forebrain (Chang et al., 2020). In the present study, we characterize the developmental expression pattern of Zswim6 mRNA in the mouse forebrain. Unlike Zswim5 that is enriched in the medial ganglionic eminence (MGE) of the ventral forebrain where progenitors of cortical and striatal interneurons reside (Marin et al., 2000), Zswim6 is highly expressed in the adjacent structure of lateral ganglionic eminence (LGE) where progenitors of striatal projection neurons are located (Olsson et al., 1995), which is in agreement with previous studies (Lein et al., 2007; Chang, 2009; Tischfield et al., 2017; Mayer et al., 2018). Concerning temporal regulation, in contrast to the downregulation of Zswim5 in the postnatal brain, Zswim6 expression is persistently expressed in the postnatal forebrain. Therefore, two paralogues of Zswim5 and Zswim6 have distinct spatiotemporal expression patterns in the developing mouse forebrain, which may endow them with different neurobiological functions.

\section{MATERIALS AND METHODS}

\section{Animals and Sample Collection}

ICR mice (BioLASCO Inc., Taiwan) were used in this study. All the experimental mice were bred in the Animal Center of National Yang Ming Chiao Tung University, and the experimental methods were approved by the Institutional Animal Care and Use Committee. To mark the developmental stages of mice, noon of the day observed vaginal plug in the pregnant dam was defined as embryonic day 0.5 (E0.5) of embryos. The day pups were delivered, was defined as postnatal day $0(\mathrm{P} 0)$. The brains of ICR mice at E11.5, E12.5, E13.5, E15.5, E17.5, P0, P7, P14-15, and adults were harvested with the following protocols. Time-pregnant dams were deeply anesthetized with i.p. injection of sodium pentobarbital, and the heads of embryos were immediately fixed with $4 \%$ paraformaldehyde (PFA) in $0.1 \mathrm{M}$ phosphate-buffered saline (PBS, pH 7.4) at $4^{\circ} \mathrm{C}$ overnight. For collecting postnatal brain, mice were perfused with $0.9 \% \mathrm{NaCl}$ followed by $4 \%$ PFA/PBS transcardially. After postfixation overnight, brains were cryoprotected by $30 \%$ sucrose in PBS at $4^{\circ} \mathrm{C}$ for two nights. All brains were immediately frozen with dry ice and then stored at $-70^{\circ} \mathrm{C}$ before cryostat sectioning. For cryosectioning, frozen brains were embedded in the Cryo-Gel (Instrumedics) and were instantly frozen with dry ice. The brains were sectioned into $20 \mu \mathrm{m}$ or $25 \mu \mathrm{m}$ with a cryostat (Leica CM1900). After sectioning, the slides were air-dried in a desiccator connected with a vacuum pump for a few hours and stored at $-20^{\circ} \mathrm{C}$.

\section{Subcloning of Zswim6 Probes for In situ Hybridization}

Zswim6 cDNA fragment with a truncated $\mathrm{N}$-terminal subcloned within $\mathrm{pBC}$ SK+ vector (mKIAA1577) was kindly provided by the Kazusa organization in Japan. The truncated Zswim6 cDNA fragment $(4,218 \mathrm{bp})$ was inserted into multiple cloning sites of the Chloramphenicol resistance $\mathrm{pBC} S \mathrm{SK}+$ vector $(3,400 \mathrm{bp})$ between the XhoI and NotI site. The mKIAA1577 was first digested with restriction enzyme SpeI (NEB) at $37^{\circ} \mathrm{C}$ overnight, generating two fragments long $1,245 \mathrm{bp}$ and $6,375 \mathrm{bp}$. The 6,375 bp DNA fragment was then eluted out by elution kit (Geneaid) and digested with NotI (NEB) at $37^{\circ} \mathrm{C}$ overnight. After enzyme digestion, the $1,331 \mathrm{bp}$ target fragment originally located between the SpeI site (NEB) at 2,888 bp and NotI site (NEB) at 4,218 bp of the mKIAA1577 sequence was generated. In the meantime, pBluescript SK (-) was also digested to form 
the 2,983 bp targeting vector by double enzyme digestion using SpeI (NEB) and NotI (NEB) at $37^{\circ} \mathrm{C}$ overnight. The $1,331 \mathrm{bp}$ fragment of mKIAA1577 was then ligated with the 2,983 bp pBluescript SK (-) targeting vector by T4 ligase (NEB) at $16^{\circ} \mathrm{C}$ overnight. The ligation products were transformed using DH5 $\alpha$ (Yeastern Biotech) and selected by antibiotics. Clones with correct ligation were identified by PCR using T3 and T7 primers with the following PCR condition: $94^{\circ} \mathrm{C}$ for $3 \mathrm{~min}$, 30 cycles of denaturation $\left(94^{\circ} \mathrm{C}\right.$ for $30 \mathrm{~s}$, annealing $\left(55^{\circ} \mathrm{C}\right.$ for $30 \mathrm{~s}$, and extension $\left(72^{\circ} \mathrm{C}\right.$ for $\left.2 \mathrm{~min}\right), 72^{\circ} \mathrm{C}$ for $2 \mathrm{~min}$, and finally stopped at $4^{\circ} \mathrm{C}$. The positive clones were further checked with restriction enzymes and PstI (NEB) and EcoRV (NEB) to generate fragments of 1,273 bp; 3,058 bp; $275 \mathrm{bp}$ and 4,056 bp respectively. Another Zswim6 riboprobe was generated by PCR cloning according to the previous study (Tischfield et al., 2017).

\section{Synthesis of Digoxigenin (Dig)-Labeled Riboprobes}

The Zswim6 riboprobes were generated as described above. The rat Drd1 and Drd2L riboprobes were kindly provided by K. Kobayashi at Fukushima Medical University, Fukushima, Japan. The riboprobes were synthesized by in vitro transcription with digoxigenin (dig) or fluorescein (FITC) RNA labeling mix (Roche). Briefly, respective linearized template plasmid was mixed with $5 \times$ transcription buffer, $0.1 \mathrm{M}$ dithiothreitol (DTT), Dig-labeling mix (Roche), RNasin (Promega), respective RNA polymerase (T3, T7, SP6, Promega), and DEPC-treated $\mathrm{H}_{2} \mathrm{O}$ at $37^{\circ} \mathrm{C}$ for $2 \mathrm{~h}$ according to manufacturer's instruction (Promega). The DNA template was digested with DNase RQ1 at $37^{\circ} \mathrm{C}$ for $30 \mathrm{~min}$. After stopping polymerase reaction by adding $0.2 \mathrm{M}$ EDTA ( $\mathrm{pH}$ 8.0) and placed on ice for $5 \mathrm{~min}$, STE buffer $(0.1 \mathrm{M}$ $\mathrm{NaCl}, 10 \mathrm{mM}$ Tris-HCl, $\mathrm{pH}$ 8.0; 1 mM EDTA, $\mathrm{pH}$ 8.0) and $3 \mu \mathrm{l}$ 1 M DTT were added. Finally, the probes were further purified with G-50 mini Quick Spin Columns (Roche). The size and quality of probes had been checked by gel electrophoresis before DNase RQ1 treatment and after purification.

\section{Digoxigenin-Labeled In situ Hybridization}

Slides stored at $-20^{\circ} \mathrm{C}$ were first air-dried at room temperature (RT) for $10 \mathrm{~min}$ and then further dried in the desiccator connected with a vacuum pump. After stopping polymerase reaction by adding $0.2 \mathrm{M}$ EDTA ( $\mathrm{pH} 8.0$ ) and being placed on ice for $5 \mathrm{~min}$, STE buffer $(0.1 \mathrm{M} \mathrm{NaCl}, 10 \mathrm{mM}$ Tris- $\mathrm{HCl}, \mathrm{pH}$ 8.0; $1 \mathrm{mM}$ EDTA, $\mathrm{pH}$ 8.0) and $3 \mu \mathrm{l} 1 \mathrm{M}$ DTT were added. Finally, the probes were further purified with G-50 mini Quick Spin Columns (Roche). The size and quality of probes had been checked by gel electrophoresis before DNase RQ1 treatment and after purification for more than $1 \mathrm{~h}$. Embryonic sections were washed in $0.01 \mathrm{M}$ PBS for $5 \mathrm{~min}$ and treated with $0.1 \%$ Triton X-100 in $0.01 \mathrm{M}$ PBS for $5 \mathrm{~min}$. Postnatal sections were post-fixed in $4 \%$ PFA/PBS for $30 \mathrm{~min}$ on ice and then treated with $0.3 \%$ Triton X-100 in $0.01 \mathrm{M}$ PBS for $15 \mathrm{~min}$. After washing with $0.01 \mathrm{M}$ PBS, all sections were incubated in $0.2 \mathrm{~N} \mathrm{HCl}$ in DEPC-treated $\mathrm{H}_{2} \mathrm{O}$ for $20 \mathrm{~min}$. Sections were treated with proteinase $\mathrm{K}(\mathrm{PK}, 10 \mu \mathrm{g} / \mathrm{ml}, \mathrm{MDBio})$ in $0.01 \mathrm{M} \mathrm{PBS}$ at $37^{\circ} \mathrm{C}$ for 2-5 min. After washing in 0.01 M PBS, sections were fixed with $4 \%$ PFA/0.01 M PBS for $5 \mathrm{~min}$ and treated with glycine $(2 \mu \mathrm{g} / \mathrm{ml})$ in $0.01 \mathrm{M}$ PBS for $15 \mathrm{~min}$ twice. Sections were then prehybridized with $50 \%$ deionized formamide (Sigma) in $2 \times$ standard saline citrate (SSC, $300 \mathrm{mM} \mathrm{NaCl}, 30 \mathrm{mM}$ sodium citrate, $\mathrm{pH} \mathrm{7.0)}$ at $65^{\circ} \mathrm{C}$ for $90 \mathrm{~min}$ in a humid oven. Diluted probes ranging from $1: 250$ to $1: 1,000$ in the hybridization solution (50\% formamide; $10 \%$ dextran sulfate; $0.3 \mathrm{M} \mathrm{NaCl} ; 0.01 \mathrm{M}$ Tris, $\mathrm{pH} 8.0 ; 500 \mu \mathrm{g} / \mathrm{ml}$ yeast tRNA; $10 \mathrm{mM}$ DTT; $1 \mathrm{mM}$ EDTA, $\mathrm{pH} 8.0$, and $1 \times$ Denhardt's solution) were denatured at $90^{\circ} \mathrm{C}$ for $10 \mathrm{~min}$, and applied onto the samples. After hybridization at $65^{\circ} \mathrm{C}$ for $16 \mathrm{~h}$, sections were washed with $5 \times$ SSC for $5 \mathrm{~min}$ and then incubated in $50 \%$ formamide (Sigma) $/ 2 \times$ SSC for $1 \mathrm{~h}$. Before and after treating with RNase $\mathrm{A}(20 \mu \mathrm{g} / \mathrm{ml})$ at $37^{\circ} \mathrm{C}$ for $30 \mathrm{~min}$, sections were incubated in $10 \mathrm{mM}$ Tris- $\mathrm{HCl}$ ( $\mathrm{pH} 8.0)$ and $500 \mathrm{mM}$ $\mathrm{NaCl}$ for $10 \mathrm{~min}$, respectively. The sections were then washed with $2 \times$ SSC once, $0.2 \times$ SSC twice for $20 \mathrm{~min}$ at $65^{\circ} \mathrm{C}$ and TNT buffer (150 mM NaCl, $100 \mathrm{mM}$ Tris $\mathrm{pH} 7.5$ ) for $10 \mathrm{~min}$ sequentially. After blocking the sections [2\% blocking reagent (Roche) and 20\% sheep serum in TNT buffer] for $60 \mathrm{~min}$, alkaline phosphatase (AP)-conjugated sheep anti-digoxigenin antibody (1:1,000, Roche, RRID:AB_514497) were incubated for 90 min. After washing the sections in TNT buffer and alkaline buffer (100 mM Tris- $\mathrm{HCl} \mathrm{pH} 9.5,100 \mathrm{mM} \mathrm{NaCl})$, signals were detected by colorimetric procedure with 5-Bromo-4-chloro-3indolyl phosphate (BCIP, Roche) and Nitro blue tetrazolium chloride (NBT, Roche) in the alkaline buffer. For the fluorescent in situ hybridization, all TNT buffer contained an additional $0.1 \%$ Tween-20. Sections were washed with $0.1 \% \mathrm{H}_{2} \mathrm{O}_{2}$ in TNT. After the blocking procedures described above, horseradish peroxidase (HRP)-conjugated sheep anti-digoxigenin (1:100, Roche; RRID:AB_514500) were incubated overnight. On the next day, the signals were detected by $\mathrm{Cy} 3$ conjugated tyramide [1:1,000 in dilution buffer, tyramide signal amplification (TSA, PerkinElmer)] for $10 \mathrm{~min}$. The fluorescent signals were analyzed with fluorescence microscopy (Eclipse E800M, Nikon; BX51, Olympus) or confocal microscopy (SP2, Leica; LSM880, Zeiss).

\section{Dual In situ Hybridization}

Following in situ hybridization protocols described above, sheep anti-FITC antibody (1:100, Roche, RRID:AB_840257) was used to identify the first type of transcript. After detection of FITC signals, the HRP activity for detecting the first antibody was bleached by $0.1 \% \mathrm{H}_{2} \mathrm{O}_{2}$ in TNT buffer for $15 \mathrm{~min}$. Then the sections were again incubated in $2 \%$ blocking solution [2\% blocking reagent (Roche), 20\% sheep serum in the TNT buffer] for an additional $60 \mathrm{~min}$. Finally, sheep anti-Dig antibody (1:100, Roche, RRID:AB_514500) was used to detect the second transcript target with overnight incubation. On the next day, the TSA system was applied with a different fluorescence according to the manufacturer's instruction.

\section{Radioactive In situ Hybridization}

After air-drying in the desiccators for over $60 \mathrm{~min}$, the sections were post-fixed with $10 \%$ formaldehyde in potassium PBS (KPBS, $1.5 \mathrm{M} \mathrm{NaCl}, 0.03 \mathrm{M} \mathrm{KH}_{2} \mathrm{PO}_{4}$, and $0.2 \mathrm{M} \mathrm{K}_{2} \mathrm{HPO}_{4}$ ) and followed by treatment with $10 \mu \mathrm{g} / \mathrm{ml} \mathrm{PK}$ in buffer containing $0.1 \mathrm{M}$ Tris ( $\mathrm{pH} 8.0$ ), and 0.05 M EDTA ( $\mathrm{pH} \mathrm{8.0)} \mathrm{for} 20 \mathrm{~min}$ at $37^{\circ} \mathrm{C}$. After rinsing in DEPC-treated $\mathrm{H}_{2} \mathrm{O}$ for $3 \mathrm{~min}$, the sections 
were incubated for $10 \mathrm{~min}$ in $0.1 \mathrm{M}$ Triethanolamine solution containing $0.25 \%$ acetic anhydride (TEA, $\mathrm{pH} 8.0$ ), and were then washed by $2 \times$ SSC buffer. After dehydration with successive rinses of ethanol $(50 \%, 70 \%, 95 \%$, and $100 \%$ twice, each for $3 \mathrm{~min}$ ), the sections were air-dried for $2 \mathrm{~h}$ and further hybridized with ${ }^{35} \mathrm{~S}$-labeled antisense probes for $16 \mathrm{~h}$ at $58^{\circ} \mathrm{C}$. The probe was mixed with the hybridization solution $\left(10^{7}\right.$ counts $\left./ \mathrm{min} / \mathrm{ml}\right)$. Before applying the hybridization mixture onto the sections, the probes were denatured for $5 \mathrm{~min}$ at $65^{\circ} \mathrm{C}$. On the next day, the sections were first washed with $4 \times$ SSC 7 min four times and were treated with $10 \mu \mathrm{g} / \mathrm{ml}$ RNase A in buffer containing $0.5 \mathrm{M}$ $\mathrm{NaCl}, 10 \mathrm{mM}$ Tris ( $\mathrm{pH} 8.0$ ), and $1 \mathrm{mM}$ EDTA ( $\mathrm{pH} 8.0$ ) for $30 \mathrm{~min}$ at $37^{\circ} \mathrm{C}$. The sections were then washed with $2 \times$ SSC twice, $1 \times$ SSC and $0.5 \times$ SSC once (each for $5 \mathrm{~min}$ ), $0.1 \times$ SSC for $30 \mathrm{~min}$ at $50^{\circ} \mathrm{C}$, and $0.1 \times \mathrm{SSC}$ for another $5 \mathrm{~min}$. All the SSC washing buffers used above contained $1 \mathrm{mM}$ DTT. After dehydration with successive 5-min rinses in ethanol (50\%, 70\%, 95\%, and $100 \%$ twice, each for $5 \mathrm{~min}$ ) and drying in the desiccators, the sections were exposed to X-ray films for detecting ${ }^{35} \mathrm{~S}$-labeled signals by autoradiography.

\section{Immunohistochemistry}

For dual in situ hybridization and immunohistochemistry experiments, in situ hybridization was performed first. For immunohistochemistry, sections were treated with $0.2 \%$ Triton $\mathrm{X}-100$ in $0.1 \mathrm{M}$ PBS (PBST) for $10 \mathrm{~min}$ after several $0.1 \mathrm{M} \mathrm{PBS}$ washes. Then the sections were treated with $3 \% \mathrm{H}_{2} \mathrm{O}_{2}$ and $10 \%$ methanol in PBST for $5 \mathrm{~min}$. After several washes with $0.1 \mathrm{M}$ PBS, sections were blocked with $3 \%$ normal goat serum (NGS) in $0.1 \mathrm{M}$ PBS for $1 \mathrm{~h}$. Then the diluted primary antibodies in PBST containing $1 \%$ NGS and $0.1 \%$ sodium azide were applied onto the sections overnight. The primary antibodies included rabbit anti-Ki67 (1:200, Leica Biosystems, RRID:AB_442102), mouse anti-Tuj1 (1:4,000, Promega, RRID:AB_430874), rabbit anti-Nolz-1 (Ko et al., 2013), and rabbit anti-Er81 (gift of Dr. A.C. Chang at National Yang-Ming University). On the next day, after several washes with $0.1 \mathrm{M}$ PBS, sections were incubated with secondary antibodies for $1 \mathrm{~h}$. For detecting Tuj1 and Er81, DTAF conjugated donkey anti-mouse (1:250, Jackson ImmunoResearch) and FITC conjugated goat-anti-rabbit (1:250, Jackson ImmunoResearch, RRID:AB_2337972) were used, respectively. For detecting Ki67 and Nolz1, biotinylated goat anti-rabbit (1:500, Vector Laboratories, RRID:AB_2313606) was used and followed by Avidin-Biotin-peroxidase complex amplification (ABC kit, Vector). The immunoreactive signals were further amplified by the TSA system.

\section{Quantification and Statistical Analysis}

Photomicrographs of D1R;Zswim6 or D2R;Zswim6 double in situ hybridization were taken from the rostral, middle, and caudal striatum of three adult mouse brains with the aid of a confocal microscope (SP2 confocal, Leica). For each brain, confocal images were acquired from eight regions that were randomly chosen in the striatum at each anatomical level. Positive cells with good signal quality in the confocal images were counted. The total number of striatal cells that were analyzed from the three brains were as follows: Rostral level:
D1R;Zswim6, $n=1,029$ cells, D2R;Zswim6, $n=976$ cells; Middle level: D1R;Zswim6, $n=970$ cells, D2R;Zswim6, $n=984$ cells; Caudal level: D1R;Zswim6 cells, $n=394$ cells, D2R;Zswim6, $n=590$ cells. The student's $t$-test was used for statistical analysis.

\section{RESULTS}

We performed in situ hybridization using digoxigenin-labeled riboprobes to characterize the developmental expression pattern of Zswim6 transcripts in mouse forebrain from embryonic stages to adulthood. In parallel, we also performed in situ hybridization using ${ }^{35}$ S-isotope-labeled riboprobes in most developmental stages. We performed in situ hybridizations with both digoxigenin- and ${ }^{35}$ S-labeled riboprobes for two reasons. First, ${ }^{35}$ S-labeled riboprobes are presumably more sensitive than digoxigenin-labeled riboprobes in detecting low levels of Zswim6 mRNA. Second, ${ }^{35}$ S-labeled riboprobes in situ hybridization, however, does not provide spatial resolution at the cellular level, which could be resolved by digoxigenin-labeled riboprobes in situ hybridization. We, therefore, performed in situ hybridizations with digoxigenin-labeled and ${ }^{35}$ S-labeled Zswim6 riboprobes in two sets of developing brain sections. The results showed that the pattern of ${ }^{35}$ S-labeled riboprobes was generally in agreement with that of digoxigenin-labeled riboprobes in situ hybridization. These experiments with two different labeled riboprobes validated the expression pattern of Zswim6 mRNA in the developing mouse forebrain. Detailed expression patterns of Zswim6 mRNA in the mouse forebrain across developmental stages are summarized in Table $\mathbf{1}$.

\section{Zswim6 mRNA Expression Pattern in the Embryonic Mouse Forebrain}

\section{E11.5}

The expression pattern of Zswim6 mRNA in the E11.5 forebrain $(n=5)$ from rostral to caudal levels is illustrated in Figure 1. Zswim6 mRNA was highly expressed in the subventricular zone (SVZ) of lateral ganglionic eminence (LGE, striatal anlage; Figures 1A1-A4,B1-B4,C,C1). Once the progenitor cells in the SVZ migrated into the differentiated mantle zone (MZ), Zswim6 was down-regulated as indicated by weak signals in the MZ of LGE (Figures 1C,C1). Zswim6 was also expressed in the SVZ and MZ of medial ganglionic eminence (MGE) and caudal ganglionic eminence (CGE; Figures 1A2-5,B3-B6,C,C2). Low levels of Zswim6 expression were found in the ventricular zone (VZ) of the three ganglionic eminences. Zswim6 mRNA was detected in the primordium of the amygdala (Figure 1B8) and hypothalamus (Figures 1A6,B9,B10). Zswim6 expression was detected in the primordia of the retina and lens (Figure 1B6).

\section{Zswim6 mRNA Expression at E12.5 and E13.5}

The expression pattern of Zswim6 in E12.5 $(n=10)$ and E13.5 forebrain $(n=7)$ from rostral to caudal levels are illustrated in Figures 2, 3. The expression pattern of Zswim6 at E12.5 was similar to that at E13.5. Zswim6 was weakly expressed in the septum (Figures 2A1,B1, 3A1,B1). Zswim6 expression was strong in the SVZ but weak in the MZ of LGE, suggesting 
TABLE 1 | Expression pattern of Zswim6 in the developing mouse forebrain.

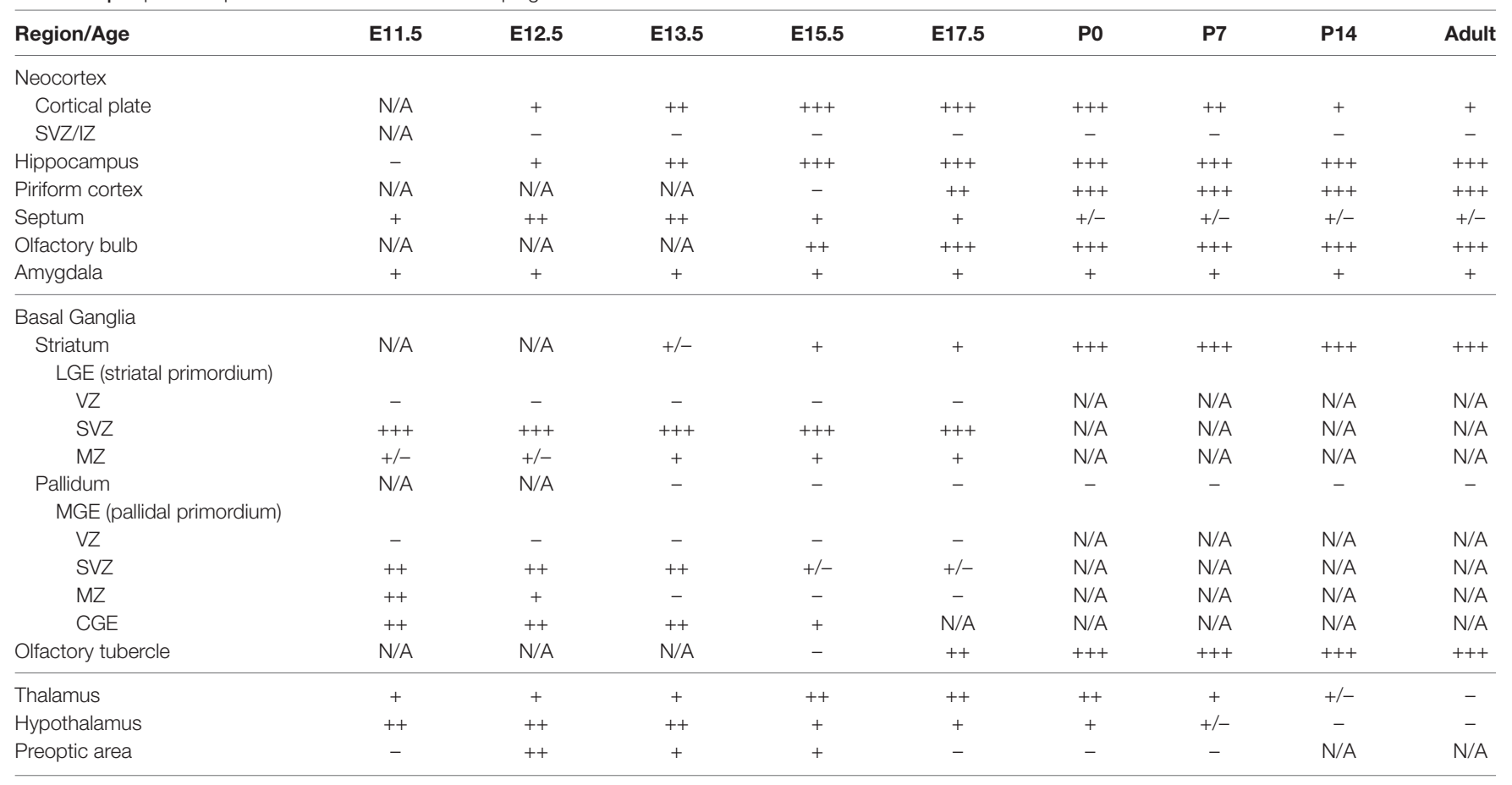

The relative expression intensity of each structure is defined compared to the structure with the strongest expression at each stage. For some structures with very weak expression identified by alkaline phosphatase signals, ${ }^{35} \mathrm{~S}$-radioactive data is evaluated for reference. The signal intensity is classified as: strong (+++), moderate (++), weak (+), very weak (+/-), or no expression (-). CGE, caudal ganglionic eminence; E, embryonic day; IZ, intermediate zone; LGE, lateral ganglionic eminence; MGE, medial ganglionic eminence; MZ, mantle zone; N/A, not applicable; P, postnatal day; SVZ, subventricular zone; VZ, ventricular zone.
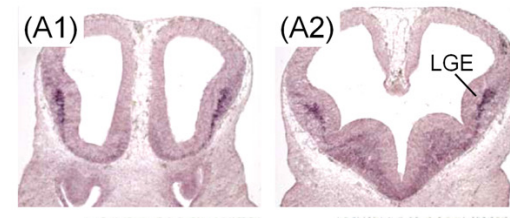

(B1)

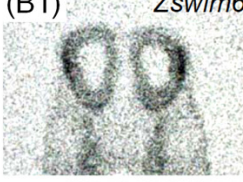

(B2)
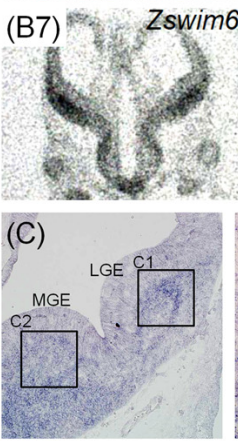
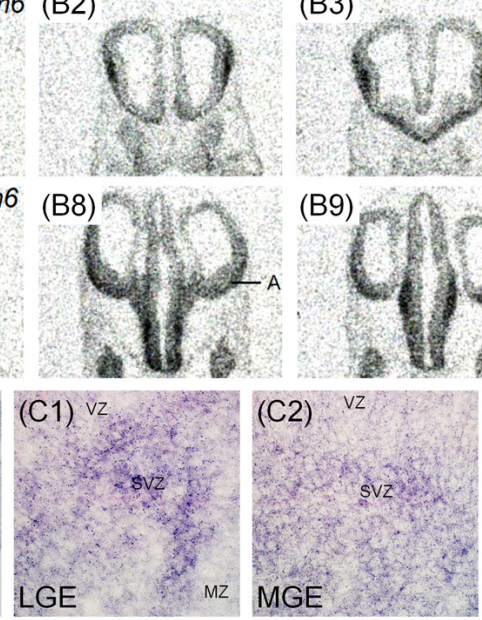

(B9)

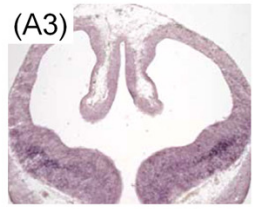

(B3)
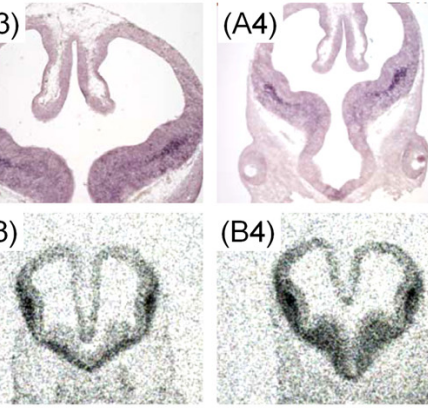

(B4)
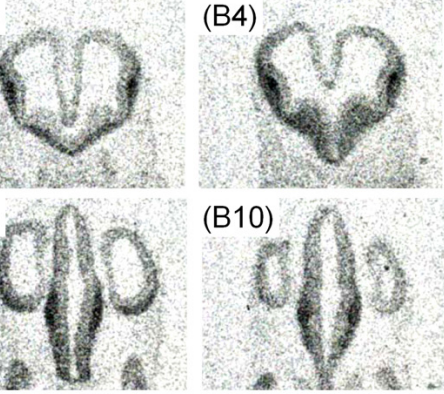

(A5)

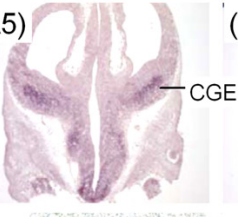

(B5)

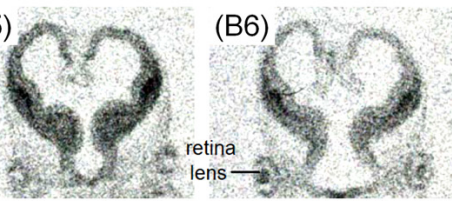

(B10)

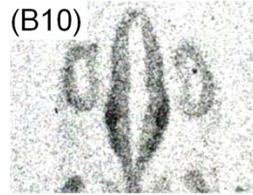

FIGURE 1 | Zswim6 mRNA expression pattern in E11.5 mouse forebrain. Expression pattern of Zswim6 mRNA analyzed by in situ hybridization with digoxigenin-labeled probes (A1-A6) and ${ }^{35}$ S-labeled probes (B1-B10) from rostral to caudal levels. (C) Zswim6 is strongly expressed in the SVZ of LGE (C,C1), but is weaker in the SVZ of MGE (C,C2). A, amygdala; CGE, caudal ganglionic eminence; LGE, lateral ganglionic eminence; MGE, medial ganglionic eminence; MZ, mantel zone; Sep, septum; SVZ, subventricular zone; VZ, ventricular zone. 
that Zswim6 expression was down-regulated in progenitor cells of the LGE while undergoing neuronal differentiation (Figures 2A2,A3,B2-B5,C,C1, 3A2-A4,B2-B5,C,C1). In the MGE and CGE, Zswim6 was also expressed in the SVZ and MZ, but not in the VZ (Figures 2A2-A5,C,C2, 3A2-A5,B2-B5,C,C2). Notably, Zswim6 expression formed a boundary at the dorsal-most part of the SVZ of LGE at E12.5 and E13.5 (Figures 2D, 3A2,A3). At caudal levels, Zswim6 expression was detected in the hypothalamus (Figures 2A5,A6,B9,B10, 3A5,A6,B7-9). Fields of Forel, one of the subdivisions of the hypothalamus, had the highest expression (Figures 3A6,B9). Zswim6 was homogenously expressed in the primordia of the retina and lens (Figures 2A3,B5, 3A2,B3).

\section{Zswim6 mRNA Expression at E15.5}

The expression pattern of Zswim6 mRNA in the E15.5 forebrain ( $n=8$ ) from rostral to caudal levels are illustrated in Figure 4. Zswim6 was strongly expressed in the early developing olfactory bulbs (OB; Figures 4A1,B1). At E15.5, strong expression of Zswim6 was maintained in the SVZ and the expression was low in the $\mathrm{MZ}$ of the developing striatum (Figures 4A2-A5). As in earlier stages, Zswim6 expression showed a boundary at the dorsal-most part of the developing striatum (Figure 4C1). Zswim6 expression was detected in the developing cortical plates (CP) and hippocampus from rostral to caudal levels (Figures 4A2-A7,B2-B9,C2). Weak expression of Zswim6 was detected in the thalamus and hypothalamus (Figures 4A6,A7,B8,B9).

\section{Zswim6 mRNA Expression at E17.5}

The expression pattern of Zswim6 mRNA in the E17.5 forebrain $(n=5)$ from rostral to caudal levels is illustrated in Figure 5. In the developing $\mathrm{OB}$, striatum, and septal area, the expression pattern of Zswim6 at E17.5 was similar to that in the E15.5 forebrain. In the developing cortex, Zswim6 expression was detected in the orbital cortex (Figure 5A1). Dorsal to the Zswim6-positive piriform cortex and olfactory tubercle, Zswim6 was expressed in the CP from rostral to caudal levels (Figures 5A2-A6,B2-B6). The cingulate cortex had strong expression (Figures 5A4,B3,B4). The expression of Zswim6 in the striatum appeared to have a dorsal to ventral decreasing gradient at the level where anterior commissure appeared (Figure 5B4). Other structures positive for Zswim6 expression included the medial habenular nucleus, the nucleus of the lateral olfactory tract, and the hippocampus (Figures 5A5,A6,B6,B7).

\section{Zswim6 mRNA Expression in Postnatal Mouse Forebrain Zswim6 mRNA Expression at P0 and P7}

The expression pattern of Zswim6 on postnatal day (P) 0 and P7 forebrain was illustrated in Figures 6, 7. Zswim6 was persistently expressed in developing OB at P0 (Figures 6A1,B1).
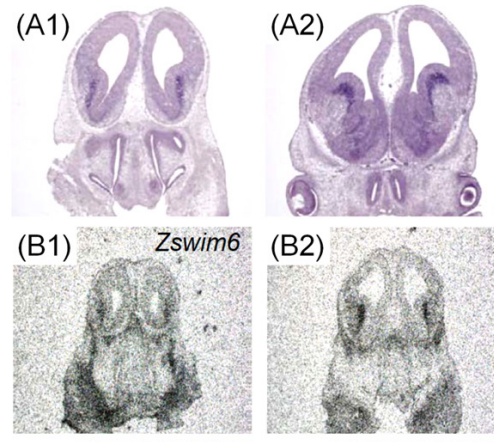

(B2)

(B7)

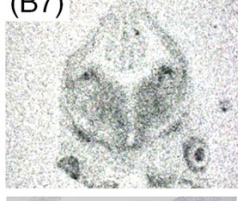

(C)

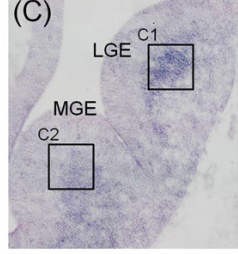

(B8)
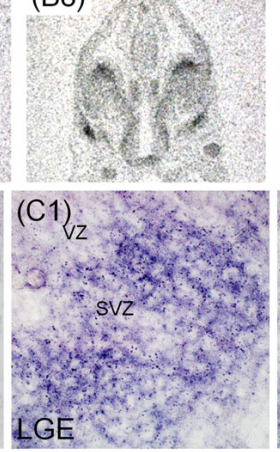

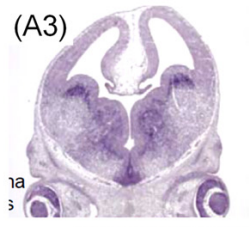

(B3)

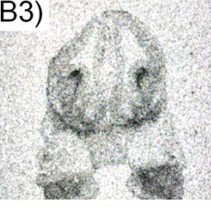

(B9)

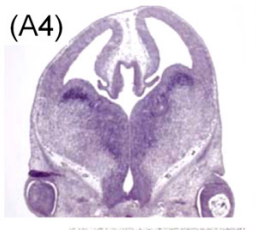

(B4)
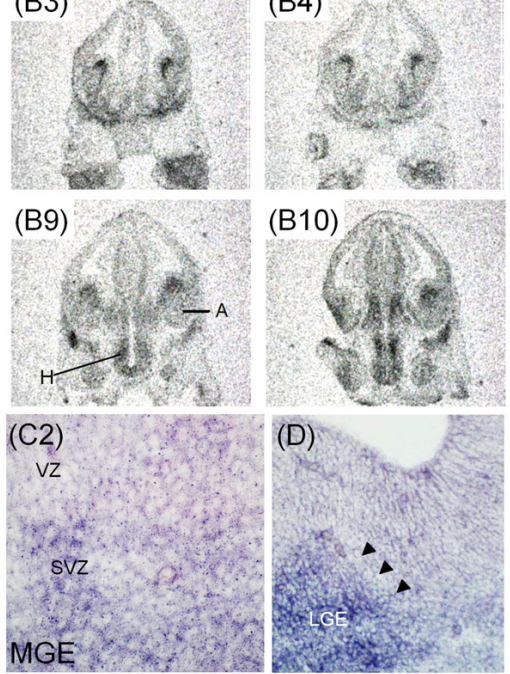

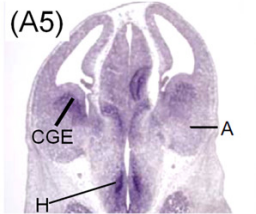

(B5)
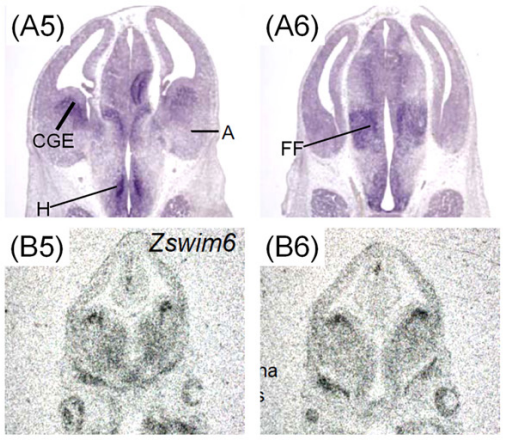

FIGURE 2 | Zswim6 mRNA expression pattern in E12.5 mouse forebrain. Expression pattern of Zswim6 mRNA analyzed by in situ hybridization with digoxigenin-labeled probes (A1-A6) and ${ }^{35}$ S-labeled probes (B1-B10) from rostral to caudal levels. (C) Zswim6 is strongly expressed in the SVZ of LGE (C,C1), but is weaker in the SVZ of MGE (C,C2). (D) Zswim6 is highly expressed in the SVZ of LGE. There is a boundary at the dorsal-most part of the SVZ of LGE (arrowheads). A, amygdala; CGE, caudal ganglionic eminence; CP, cortical plate; H, hypothalamus; Hip, hippocampus; LGE, lateral ganglionic eminence; MGE, medial ganglionic eminence; MZ, mantel zone; SVZ, subventricular zone; VZ, ventricular zone. 

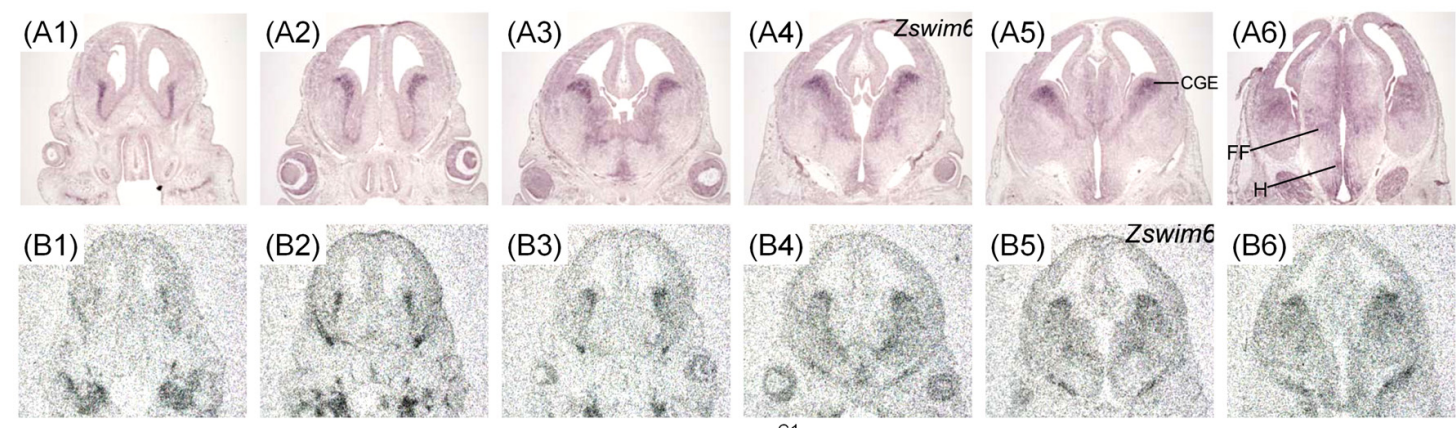

(B2)

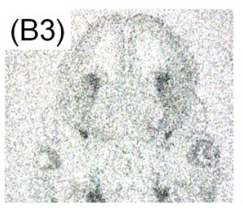

(B4)
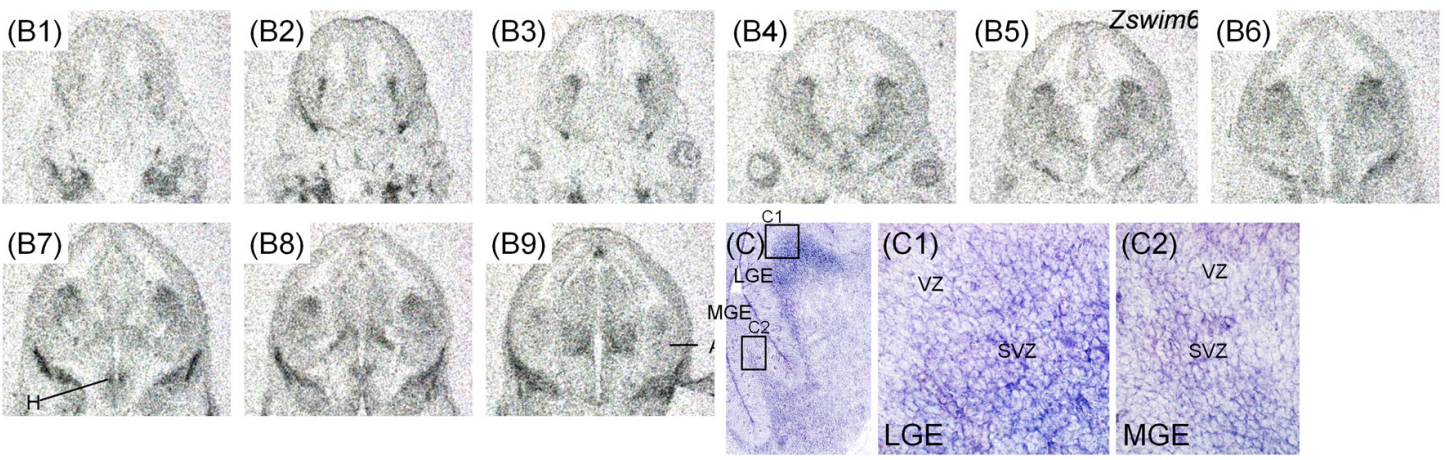

FIGURE 3 | Zswim6 mRNA expression pattern in E13.5 mouse forebrain. Expression pattern of Zswim6 mRNA detected by in situ hybridization with digoxigenin-labeled probes (A1-A6) and ${ }^{35}$ S-labeled probes (B1-B9) from rostral to caudal levels. (C) Zswim6 is strongly expressed in the SVZ of LGE (C,C1). Weak expression of Zswim6 is present in the SVZ of MGE (C,C2). A, amygdala; CGE, caudal ganglionic eminence; CP, cortical plate; FF, Fields of Forel; H, hypothalamus; LGE, lateral ganglionic eminence; MGE, medial ganglionic eminence; MZ, mantel zone; SVZ, subventricular zone; VZ, ventricular zone.
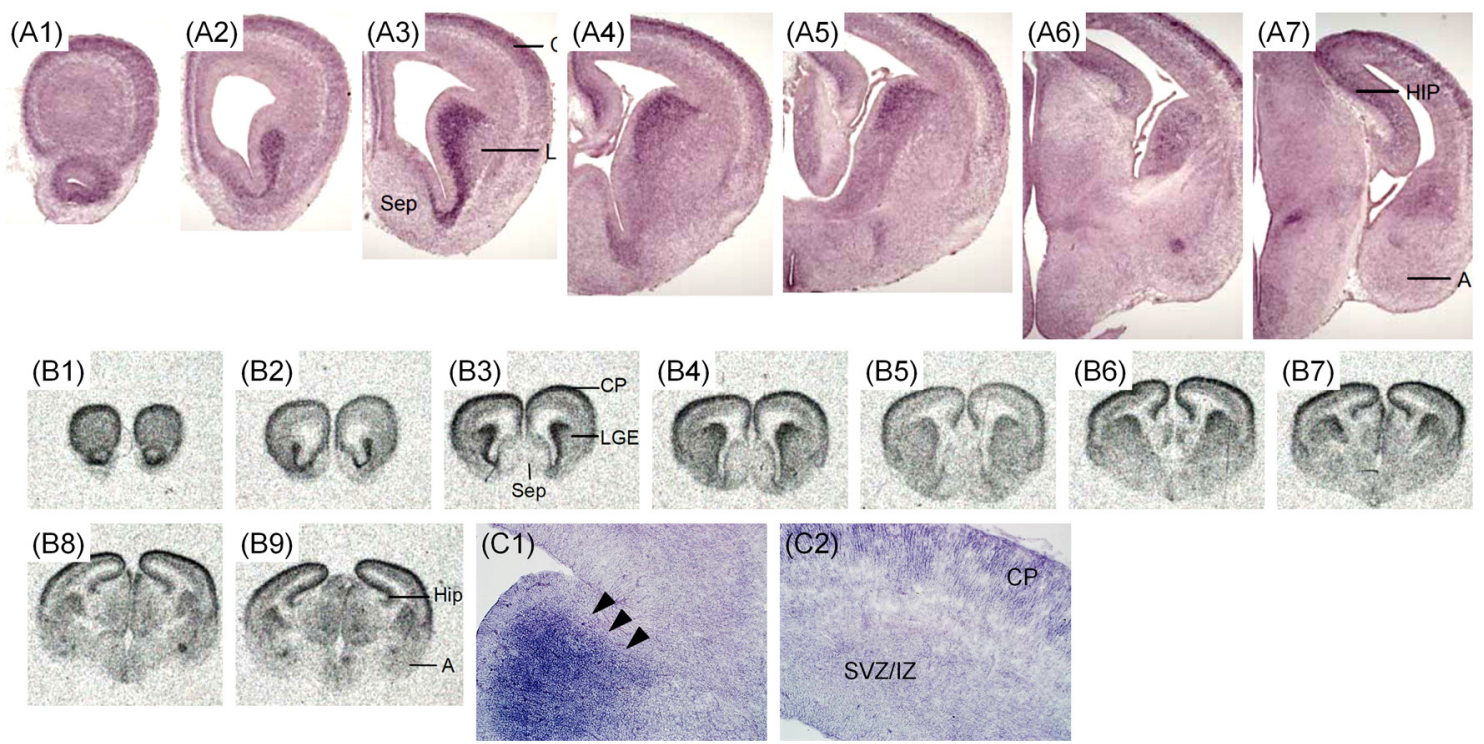

FIGURE 4 | Zswim6 mRNA expression pattern in E15.5 mouse forebrain. Expression pattern of Zswim6 mRNA detected by in situ hybridization with digoxigenin-labeled probes (A1-A7) and ${ }^{35}$ S-labeled probes (B1-B9) from rostral to caudal levels. Zswim6 is mainly expressed in the SVZ of developing striatum with a border at the dorsal-most part (arrowheads in C1). Zswim6 is expressed in the developing cortex (C2). A, amygdala; CGE, caudal ganglionic eminence; CP, cortical plate; Hip, hippocampus; IZ, intermediate zone; LGE, lateral ganglionic eminence; MGE, medial ganglionic eminence; Sep, septum; SVZ, subventricular zone; VZ, ventricular zone.

Zswim6 expression was detected in the mitral cell layer and granule cell layer of $\mathrm{OB}$ at $\mathrm{P} 7$ (Figure 7D). Zswim6 was expressed in the developing cortex (Figures 6A2-A4,B2-B4, 7F-H). The cingulate cortex in the medial cortical region was positive for Zswim6 expression (Figures 6B3,B4, 7G). Zswim6 signals in the cortex were further expended ventrally into the piriform cortex, olfactory tubercle and if any, the islands of Calleja
(Figures 6A3,A4,B3,B4, 7A,B,I). In the striatum, Zswim6 was persistently expressed in $\mathrm{P} 0$ and $\mathrm{P} 7$ dorsal striatum as well as in the ventral striatum, including the nucleus accumbens $(\mathrm{Acb})$ core $(\mathrm{AcbC})$ and shell (AcbS; Figures 6A3,A4,B2-B6, 7A-C,I). The signal intensity and expressing cell number appeared to be lower in the AcbS than that in the AcbC (Figures 6B3, 7I). A dorsal to ventral decreasing gradient of Zswim6 expression was observed 

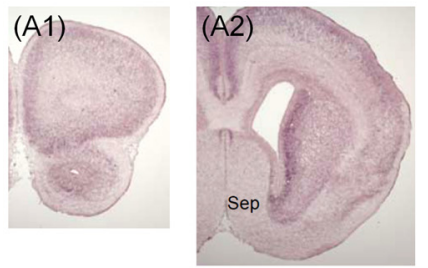

(B1)

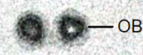

(B2)
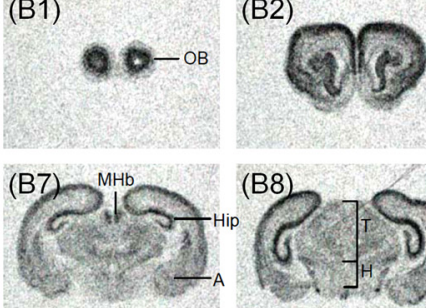
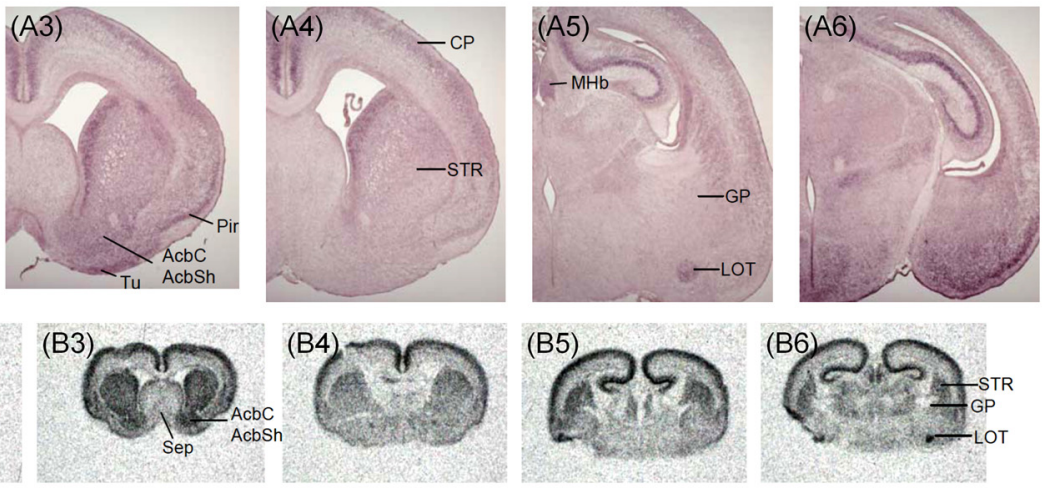

FIGURE 5 | Zswim6 mRNA expression pattern in E17.5 mouse forebrain. Expression pattern of Zswim6 mRNA detected by in situ hybridization with digoxigenin-labeled probes (A1-A6) and ${ }^{35}$ S-labeled probes (B1-B9) from rostral to caudal levels. A, amygdala; AcbC, nucleus accumbens, core; AcbSh, nucleus accumbens, shell; CP, cortical plate; GP, globus pallidus; H, hypothalamus; HIP, hippocampus; IZ, intermediate zone; LOT, nucleus of the lateral olfactory tract; MGE, medial ganglionic eminence; MHb, medial habenular nucleus; Pd, pallidum primordium; Pir, piriform cortex; POA, preoptic area; Sep, septum; SVZ, subventricular zone; T, thalamus; Tu, olfactory tubercle.

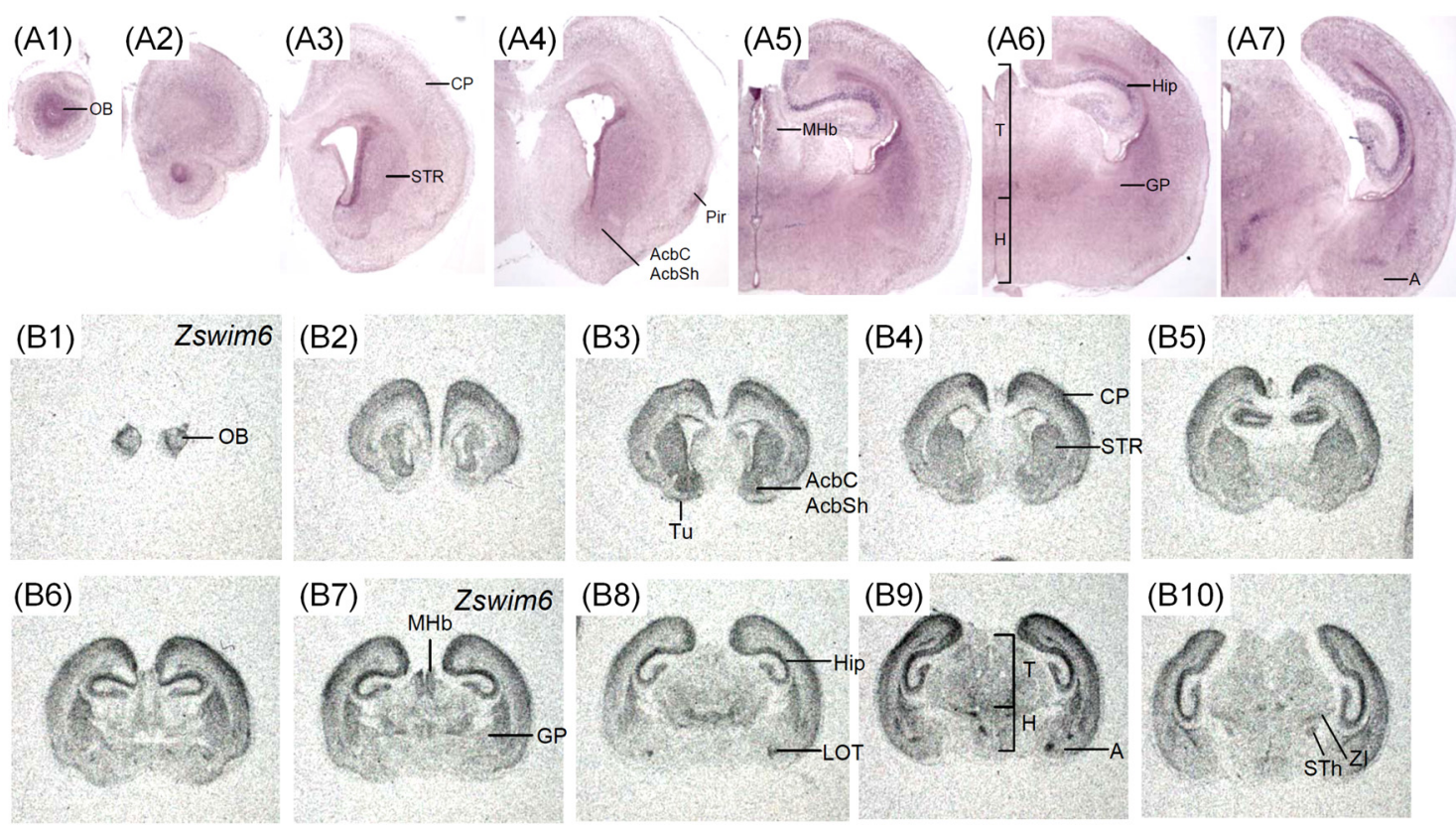

FIGURE 6 | Zswim6 mRNA expression pattern in P0 mouse forebrain. Zswim6 mRNA signals were detected by in situ hybridization with digoxigenin-labeled probes (A1-A7) and ${ }^{35}$ S-labeled probes (B1-B10) from rostral to caudal levels at P0. Moderate to strong Zswim6 signals were expressed in the olfactory bulb, dorsal and ventral striatum, cortex, hippocampus, thalamus, amygdala and medial habenular nucleus. A, amygdala; AcbC, nucleus accumbens, core; AcbSh, nucleus accumbens, shell; AO, anterior olfactory nucleus; Cere, cerebellum; Cg, cingulate cortex; CP, cortical plate; CTX, cortex; DG, dentate gyrus; GP, globus pallidus; $\mathrm{GrO}$, granular cell layer of the olfactory bulb; $\mathrm{H}$, hypothalamus; Hip, hippocampus; MHb, medial habenular nucleus; Mi, mitral cell layer of the olfactory bulb; ne, neuroepithelium; OB, olfactory bulb; Pir, piriform cortex; T, thalamus; Tu, olfactory tubercle; STh, subthalamic nucleus; STR, striatum; ZI, zona incerta.

at the middle level of the striatum (Figures 6A4,B4, 7B). In the hippocampus, Zswim6 was most strongly expressed in the pyramidal cell layer, but the weak expression was present in the granular layer of the dentate gyrus (Figures 6A5-A7,B6-B10,
7J-L). Other regions expressing Zswim6 included the medial habenular nucleus, thalamus, zona incerta, subthalamic nucleus, the nucleus of the lateral olfactory tract, and the lateral or basolateral amygdaloid nucleus (Figures 6A5-A7,B7-B10, 


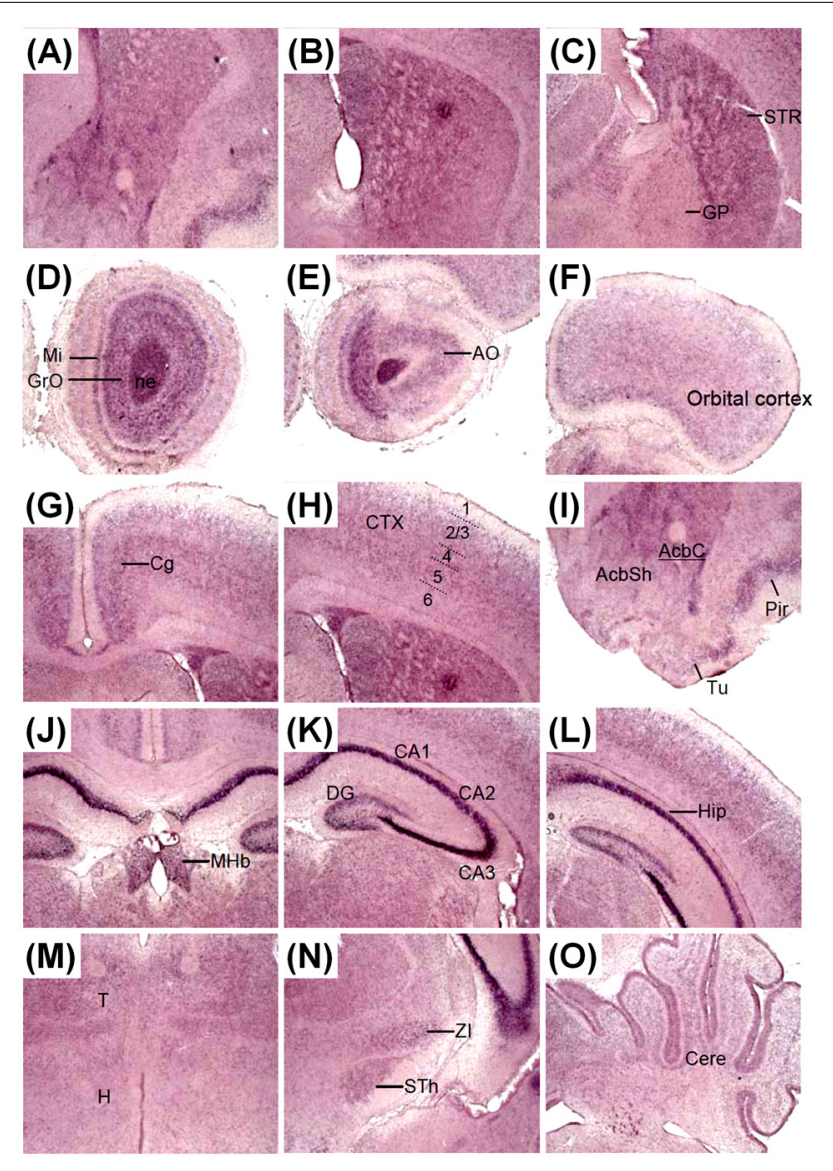

FIGURE 7 | Zswim6 mRNA expression pattern in P7 mouse forebrain. Zswim6 mRNA signals were detected from rostral to caudal levels at P7 (A-C). Moderate to strong Zswim6 signals were expressed in the olfactory bulb $(\mathbf{D}, \mathbf{E})$, dorsal and ventral striatum $(\mathbf{A}-\mathbf{C}, \mathbf{I})$, cortex (F-I), hippocampus $(\mathbf{J}-\mathbf{L})$, thalamus $(\mathbf{M}, \mathbf{N})$, cerebellum $(\mathbf{O})$, and medial habenular nucleus $(\mathbf{J})$. AcbC, nucleus accumbens, core; AcbSh, nucleus accumbens, shell; AO, anterior olfactory nucleus; Cere, cerebellum; Cg, cingulate cortex; CP, cortical plate; CTX, cortex; DG, dentate gyrus; GP, globus pallidus; GrO, granular cell layer of the olfactory bulb; $\mathrm{H}$, hypothalamus; Hip, hippocampus; $\mathrm{MHb}$, medial habenular nucleus; Mi, mitral cell layer of the olfactory bulb; OB, olfactory bulb; Pir, piriform cortex; T, thalamus; Tu, olfactory tubercle; STh, subthalamic nucleus; STR, striatum; ZI, zona incerta.

7K-N). Zswim6 expression in the thalamus and hypothalamus was down-regulated at P0 and P7 (Figures 6A6,B9, 7J).

\section{Zswim6 mRNA Expression at P14 and Adulthood}

The expression pattern of Zswim6 in the P14 forebrain $(n=3$; Figure 8) was similar to that in the P7 forebrain. However, the expression levels of Zswim6 in the cerebral cortex and the thalamus appeared to be down-regulated at P14 compared to earlier stages (Figure 8A7). In the adult forebrain, persistent expression of Zswim6 was detected in the $\mathrm{OB}$, striatum, AcbC, AcbS, pyramidal cell layer of the hippocampus, granule cell layer of the dentate gyrus, olfactory tubercle, piriform cortex, medial habenular nucleus, and the substantia nigra (Figure 8). In the adult brain, a lateral-to-medial increasing gradient of Zswim6 in the striatum was observed in parasagittal sections

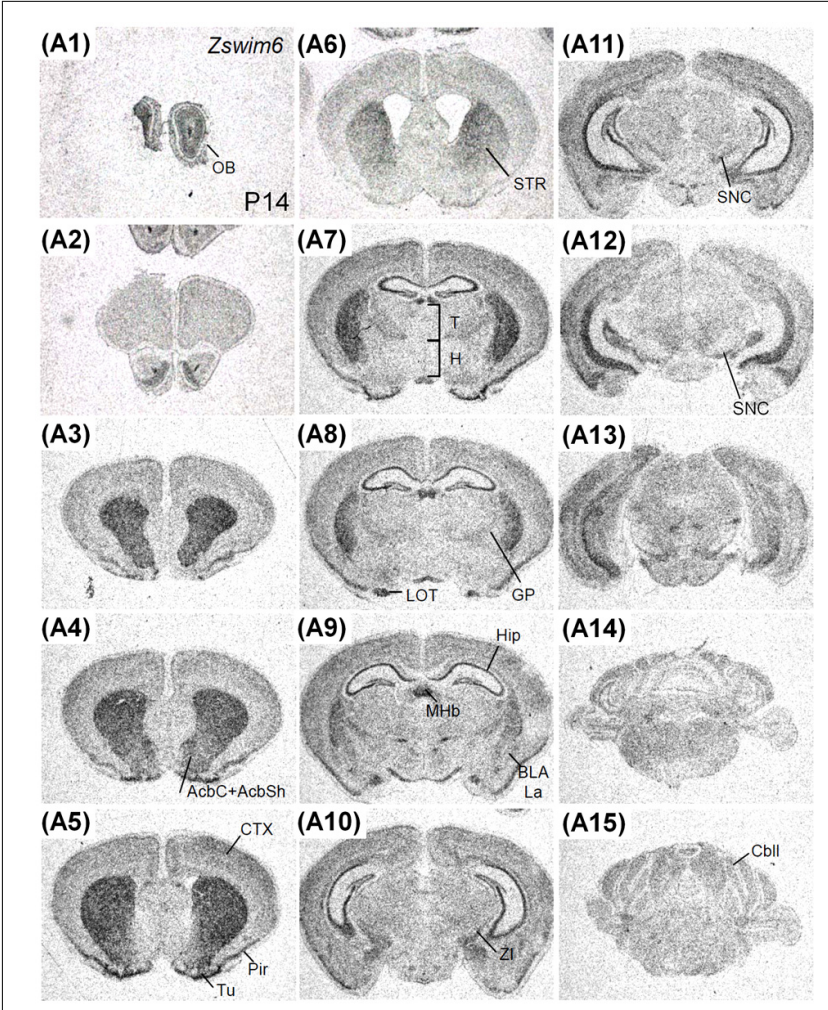

FIGURE 8 | Zswim6 mRNA expression pattern in P14 mouse forebrain. Zswim6 mRNA signals were detected from rostral to caudal levels at P14 (A1-A15). AcbC, nucleus accumbens, core; AcbSh, nucleus accumbens, shell; BLA, basolateral amygdala; Cbll, cerebellum; CTX, cortex; GP, globus pallidus; $H$, hypothalamus; Hip, hippocampus; La, lateral amygdaloid nucleus; LOT, nucleus of the lateral olfactory tract; $\mathrm{MHb}$, medial habenular nucleus; OB, olfactory bulb; Pir, piriform cortex; SNC, substantia nigra; STR, striatum; T, thalamus; Tu, olfactory tubercle; ZI, zona incerta.

(Figures 9A1-A6). Zswim6 expression in the cortex was lower than that in the striatum in adulthood (Figures 9A1-A6).

\section{Cell-Type Analysis of Zswim6 Expression}

\section{Zswim6 Is Expressed in Nolz1-Positive but Er81-Negative Differentiating Progenitors of LGE}

To determine whether Zswim6 was expressed in the population of proliferating or differentiating progenitors, Zswim6 mRNA was double-labeled with the proliferating marker of Ki67 or the differentiating marker of Tuj1 in the E13.5 forebrain (Figures 10A1-A4,B1-B5). In the SVZ of LGE, Zswim6positive cells express none or at most low levels of Ki67, and cells expressing high levels of Ki67 contained none or low levels of Zswim6 (Figures 10A1-A4). By contrast, Tuj1-positive cells were found to co-express Zswim6 in the SVZ of LGE (Figures 10B1-B4). Moreover, in the differentiated MZ of LGE, which contained high levels of Tuj1 but low levels of Zswim6, Tuj1 was also colocalized with Zswim6 (Figure 10B5). These findings indicated that Zswim6-positive cells in the SVZ of LGE were postmitotic cells undergoing early stages of neuronal differentiation in the developing striatum. 


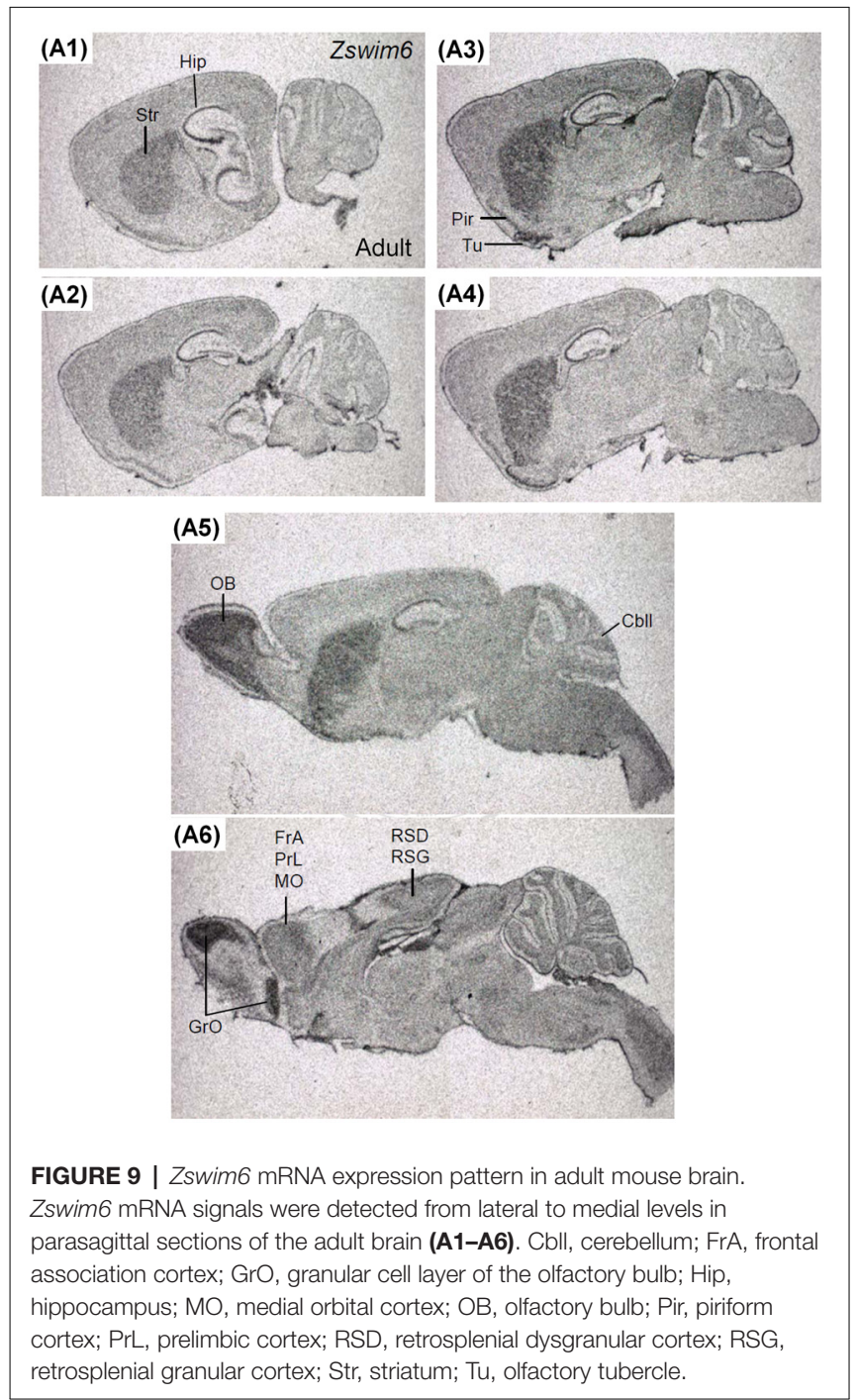

Nolz-1 is a marker of differentiating progenitors of striatal projection neurons (Chang et al., 2004). Similar to Zswim6, Nolz-1 is also highly expressed in the SVZ of E13.5 LGE. We determined whether Zswim6 and Nolz-1 were expressed in the same progenitor population. The double in situ hybridization showed that Zswim6 and Nolz-1 mRNA expression domains overlapped in the SVZ of LGE except the dorsal-most part of LGE where Nolz-1 expression was absent (Figures 10C1-C3). At the single-cell level, co-localization of Zswim6 and Nolz1 was found in the progenitors of SVZ (Figure 10C5), but not in the progenitors of the dorsal-most region of LGE (Figure 10C4). These findings indicated that, except for cells in the dorsal part of LGE, Zswim6 and Nolz-1 were co-expressed by the majority of early differentiating progenitor cells of the ventral LGE.

Because Zswim6 expression showed a clear boundary at the dorsal-most part of the SVZ of LGE, we further examined whether Zswim6 was co-localized with Er81, a marker of dorsal LGE (Stenman et al., 2003). Cells expressing
Er81 did not co-express Zswim6 in the dorsal LGE at E15.5 (Figures 10D1-D4).

\section{Zswim6 Is Expressed in Both Striatonigral and Striatopallidal Neurons}

Because Zswim6 was expressed in the adult striatum, we investigated whether Zswim6 was specifically expressed in the populations of dopamine D1 receptor (D1R)-expressing striatonigral neurons and/or dopamine D2 (D2R)-expressing striatopallidal neurons. The double in situ hybridization of Zswim6 and $D 1 R$ or $D 2 R$ showed co-expression of Zswim6 and $D 1 R$ (Figures 10E1-E4) or $D 2 R$ (Figures 10F1-F4). Quantitative analysis indicated that the co-localization ratio of $D 2 R^{+} ; Z$ swim $6^{+}$cells/Zswim6 ${ }^{+}$cells was moderately higher than that of $D 1 R^{+} ; Z$ swim6 ${ }^{+}$cells/Zswim6 ${ }^{+}$cells (Figure 10G). At the rostral level, the co-expression ratios of $D 1 R^{+} ; Z$ swim $6^{+} / Z$ swim $6^{+}$ cells and $D 2 R^{+} ; Z$ swim6 ${ }^{+} / Z$ swim6 $6^{+}$cells were $58.99 \% \pm 1.72$ and $61.46 \% \pm 1.78 \%$, respectively $(p=0.3134, n=3)$. At the middle level, the co-expression ratios of $D 1 R^{+} ; Z$ swim $6^{+} / Z$ swim6 $6^{+}$cells

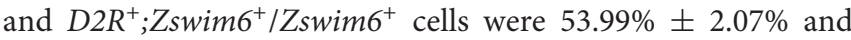
$60.29 \% \pm 1.56 \%$, respectively $(p=0.0163, n=3)$. At the caudal level, where the anterior commissure crosses the midline, the co-expression ratios of $D 1 R^{+} ; Z$ swim6 $6^{+} / Z$ swim6 $6^{+}$ cells and ${\mathrm{D} 2 \mathrm{R}^{+} ; Z \text { swim6 }}^{+} / Z_{\text {swim6 }}{ }^{+}$cells were $50.98 \% \pm 2.42 \%$ and $62.73 \% \pm 1.46 \%$, respectively $(p=0.0003, n=3)$. In brief, relative to the ratio of $D 1 R^{+} ; Z s w i m 6^{+}$cells, the co-localization ratios of $D 2 R^{+} ; Z$ swim $6^{+}$cells were $104.19 \% \pm 3.01 \%$ at the rostral level ( $p=0.3134, n=3), 111.67 \% \pm 2.89 \%$ at the middle level $(p=0.0163, n=3)$ and $123.04 \% \pm 2.86 \%$ at the caudal level $(p=0.0003, n=3)$. Therefore, Zswim6-positive neurons were moderately enriched in striatopallidal neurons than striatonigral neurons in the middle and caudal striatum at adulthood.

\section{DISCUSSION}

In the present study, we have comprehensively characterized the expression pattern of Zswim6 mRNA in the developing mouse forebrain. In the early forebrain development from E11.5 to E13.5, Zswim6 mRNA was highly expressed in the SVZ of LGE (striatal anlage). At E15.5 and E17.5, Zswim6 expression was detected not only in the developing striatum but also in other brain regions, including the developing cerebral cortex, hippocampus, medial habenular nucleus, and olfactory bulb. Zswim6 expression was persistently expressed in the postnatal brain, though the expression levels appeared to be down-regulated in the striatum and cortex.

Consistent with the previous study (Tischfield et al., 2017), Zswim6 was prominently expressed in the SVZ, but not VZ, of developing striatum. The SVZ is a transition zone from cell proliferation to differentiation. We found that most cells expressing high levels of the proliferating marker Ki67 contained none or at most low levels of Zswim6 in the SVZ. Zswim6 was co-expressed with the differentiating marker Tuj1 in the SVZ and MZ, indicating that Zswim6 is expressed in postmitotic differentiating neurons. Moreover, Zswim6 was colocalized with Nolz-1, a marker of early differentiating progenitors in the SVZ of LGE 

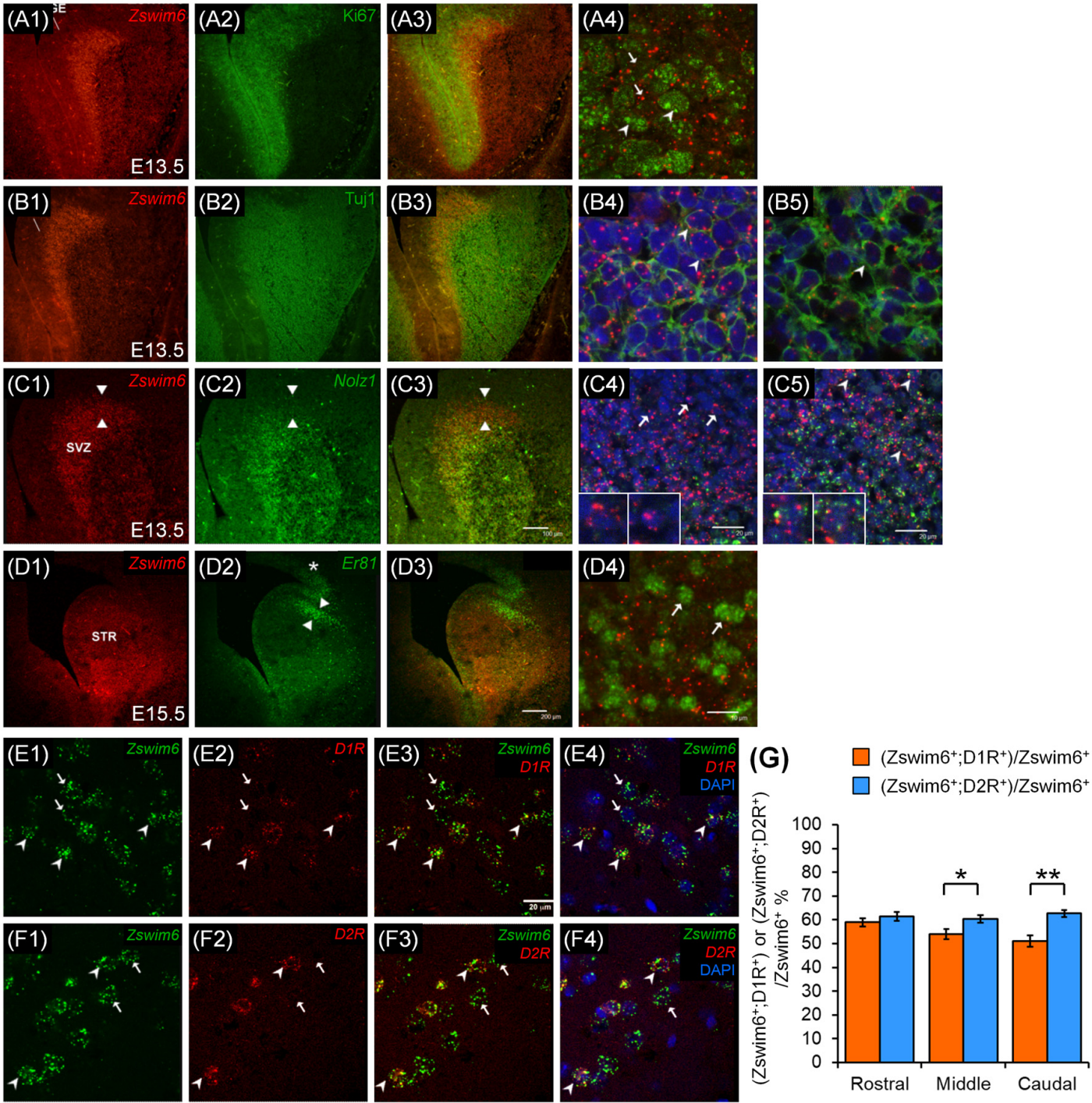

FIGURE 10 | Zswim6 are expressed in Nolz1-positive neurons in the LGE at E13.5 and striatonigral and striatopallidal neurons in adult brains. Zswim6 is mainly expressed in the SVZ of the LGE at E13.5 (A1,B1), whereas Ki67-positive (A2) and Tuj1-positive (B2) cells are located in the VZ and the MZ of the LGE, respectively. In the overlapping zone (A3), Zswim6-positive cells express none or at most low levels of Ki67 (arrows, A4). Cell expressing high levels of Ki67 contained none or low levels of Zswim6 (arrowheads, A4). Colocalization of Tuj1 protein and Zswim6 mRNA (B3) is detected in cells of SVZ (B4) and MZ (B5). (C,D) Both Zswim6 (C1) and Nolz-1 (C2) are expressed in the SVZ of LGE, and their expression domains are mostly overlapped in the ventral part of SVZ (C3). In the dorsal non-overlapping zone, cells expressing Zswim6 without Nolz-1 are present (C3, the area between arrowheads; C4, insets show high magnification of the cells indicated by arrows). In the Zswim6 and Nolz-1 overlapped regions, Zswim6 is colocalized with Nolz-1 (C5, insets show high magnification of cells indicated by arrowheads). At E15.5, Zswim6 mRNA (D1) is expressed in the SVZ of the striatal primordium, while Er81 marks the dorsal-most part of the striatal primordium (D2, between arrowheads). Although Zswim6 expression is overlapped with Er81 at the dorsal-most striatum (D3), at the single-cell level, Zswim6 mRNA signals (D4, arrows) are not co-localized with Er81-positive cells. (E,F) Confocal images of double in situ hybridization show that some Zswim6-positive cells (E1,E3,E4; arrowheads) were co-localized with D1R (E2-E4) in the mature striatum, whereas others express Zswim6 only (E1,E3,E4; arrows). In the double in situ hybridization of Zswim6 and D2R, some Zswim6-positive cells (F1,F3,F4; arrowheads) were co-localized with D2R (F2-F4) in the mature striatum, while others expressed Zswim6 only (F1,F3,F4; arrows). (G) Quantification of the co-localization ratios of Zswim6 and $D 1 R$ or $D 2 R$ in the mature striatum from the rostral to caudal levels. ${ }^{*} p<0.05$, ${ }^{* *} p<0.001, n=3$, student's $t$-test. LGE, lateral ganglionic eminence; MZ, mantle zone; STR, striatum; SVZ, subventricular zone.

(Chang et al., 2004; Ko et al., 2013; Chen et al., 2020), suggesting that Zswim6 is expressed in differentiating striatal progenitors. We further found that Zswim6 mRNA was colocalized with $D 1 R$-expressing striatonigral and $D 2 R$-expressing striatopallidal neurons of the adult striatum. Collectively, these findings indicate that Zswim6 is expressed in early differentiating progenitors, and it is persistently expressed in striatal projection neurons of the adult brain.

The SVZ of LGE contains two neuronal progenitor populations. $\mathrm{Dlx^{+ }} ; \mathrm{Is} l 1^{+}$cells make up the majority of progenitors in the ventral part of LGE that gives rise to striatal projection neurons, whereas $D l x^{+} ; E r 81^{+}$cells in the dorsal-most part of 
LGE comprises progenitors of olfactory bulb interneurons (Stenman et al., 2003). Zswim6 mRNA was mostly expressed in the ventral LGE. Double labeling of Zswim6 and Nolz-1, a marker of differentiating striatal projection neurons (Chang et al., 2004; Ko et al., 2013; Chen et al., 2020) showed that Zswim6 and Nolz-1 were colocalized in the progenitors of ventral LGE. Because Nolz-1 is co-expressed with Isl1 in early differentiating striatal projection neurons (Chang et al., 2004), and Zswim6 is co-localized with Nolz-1, Zswim6 is thus presumably expressed in $D l x^{+} ; I s l 1^{+}$progenitors of ventral LGE that gives rise to striatal projection neurons. In contrast to the high level in the ventral LGE, a low level of Zswim6 mRNA was present in the dorsal LGE in which Zswim6 was not colocalized with Er81. Therefore, Zswim6 is not expressed in $\mathrm{Dlx^{+ }} ; E \mathrm{Er} 81^{+}$progenitors of dorsal LGE where olfactory bulb interneurons reside.

Previous studies have identified several transcriptional regulators that are enriched in the LGE, MGE and CGE, e.g., Dlx1, Dlx2, Isl1, RAR $\beta$, Nolz-1, and SP9, and these transcriptional factors regulate cell-type specification, migration, and differentiation (Liao et al., 2008; Rubenstein and Campbell, 2013; Tao et al., 2019; Chen et al., 2020). Zswim6 is expressed in the LGE, MGE, and CGE and Zswim6 is up-regulated at the late progenitor state of the maturation trajectory of CGE at E14.5 (Mayer et al., 2018). The developmental expression pattern of Zswim6 suggests that Zswim6 may be involved in the neurogenesis of striatal neurons. Tischfield et al. (2017) have shown that genetic deletion of Zswim6 resulted in a moderate loss of striatal neurons but marked reductions in dendritic complexity and length, and dendritic spines of striatal neurons. No evident decreases in cell proliferation and increases in cell apoptosis were observed in the embryonic striatum of Zswim6 knockout mice (Tischfield et al., 2017). Moreover, they found no changes in the expression of several striatonigral and striatopallidal-enriched genes in Zswim6 knockout brains. Interestingly, the knockout mice are hyperactive in locomotion and they exhibit deficits in rotarod learning and repetitive motor behaviors. Notably, our present study has shown that Zswim6 is expressed at a higher level in $D 2 R$-expressing striatopallidal neurons than $D 1 R$ expressing striatonigral neurons, which is in agreement with the previous FACS-array study (Lobo et al., 2006), suggesting that Zswim6 may regulate striatal dopamine neurotransmission. Although $D 1 R$ and $D 2 R$ mRNA levels were not altered in the striatum of Zswim6 knockout mice, the knockout mice exhibited enhanced locomotion in response to dopamine agonist amphetamine (Tischfield et al., 2017). The striatum has been proposed as a key region of dopamine neurotransmission that is involved in the pathophysiology of schizophrenia (McCutcheon et al., 2019). Given that Zswim6 is associated with schizophrenia, it is of interest to investigate whether abnormal striatal dopamine neurotransmission occurs in Zswim6 knockout mice that may contribute to schizophrenia-like phenotypes.

Strong Zswim6 expression is detected in the CA1 and CA3 of the hippocampus. The hippocampus is known to be involved in the pathogenesis of schizophrenia (Ho et al., 2017; Roeske et al., 2020). Interestingly, Zswim6 is expressed in the medial habenula in which the schizophrenia-risk gene ErbB4 is also highly expressed (Steiner et al., 1999). Although the pathological role of the habenular nucleus is less characterized in schizophrenia, the habenular complex is a hub region for regulating motivation and emotion that are affected in schizophrenia (Sandyk, 1991; Namboodiri et al., 2016; Zhang et al., 2017).

Increasing evidence suggests that poorly annotated non-coding variants contribute to the pathophysiology of schizophrenia through unknown mechanisms (Takata, 2019). As non-coding regions can regulate transcription and translation of protein-coding regions, non-coding variants may cause dysregulation of protein-coding genes. Previous studies have identified overlapping mutations in different neuropsychiatric disorders, suggesting that alterations of common special regulatory elements may underlie some neuropsychiatric disorders (Hoischen et al., 2014; Li et al., 2016; Vissers et al., 2016). Bioinformatic analysis of de novo mutations from the NPdenova database has found biological pathways and non-coding regulatory elements of candidate genes that are shared among schizophrenia, autistic spectrum disorders, intellectual disability, and encephalopathy (Li et al., 2016). It would be of interest to identify non-coding variants of Zswim6 and study the non-coding regions that may regulate Zswim6 expression, which may help understand the pathology of Zswim6-associated neurological disorders.

Our previous study has characterized the expression pattern of Zswim5, another member of the Zswim family, in the developing mouse forebrain (Chang et al., 2020). In contrast to the enriched expression of Zswim6 in the LGE, Zswim5 is preferentially expressed in the adjacent structure of MGE in the ventral forebrain. ZSWIM5 is expressed in progenitors of GABAergic interneurons that tangentially migrate from the MGE to the developing cortex. Notably, in addition to the high level of expression in the LGE, Zswim6 mRNA is also expressed in the MGE as early as E11.5, suggesting that Zswim6 may also be expressed in progenitors of cortical interneurons. Zswim6 signals were detected in the developing cortex. However, it remains to be clarified whether Zswim6 is expressed in interneurons and/or projection neurons of the developing cortex. The cell type characterization of Zswim6 expression will help explore the neuronal function of Zswim6 and the pathology of Zswim6associated neurological diseases.

\section{DATA AVAILABILITY STATEMENT}

The original contributions presented in the study are included in the article, further inquiries can be directed to the corresponding author/s.

\section{ETHICS STATEMENT}

The animal study was reviewed and approved by Institutional Animal Care and Use Committee at National Yang-Ming University.

\section{AUTHOR CONTRIBUTIONS}

C-CC, H-YK, S-YC, W-TL, and K-ML performed experiments. TS provided reagents. C-CC, H-YK, and 
F-CL analyzed data and wrote the manuscript. authors contributed to the article and approved the submitted version.

\section{FUNDING}

This work was supported by the Ministry of Science and Technology-Taiwan grants MOST107-2320-B-010-041-MY3, MOST-108-2321-B-010-02, MOST-108-2321-B-007-003MY2, MOST-109-2326-B-010-001, MOST110-2321-B-001-012 (F-CL), MOST 110-2636-B-A49-001 (H-YK), Postdoctoral

\section{REFERENCES}

Chang, C. C. (2009). Characterization of the Zswim Family Genes, Zswim5 and Zswim6, in the Developing Mouse Forebrain. Master's Thesis. Taipei, Taiwan: National Yang-Ming University.

Chang, C.-C., Kuo, H.-Y., Chen, S.-Y., Lin, W.-T., Lu, K.-M., Saito, T., et al. (2020). Developmental characterization of Zswim5 expression in the progenitor domains and tangential migration pathways of cortical interneurons in the mouse forebrain. J. Comp. Neurol. 14, 2404-2419. doi: 10.1002/cne.24900

Chang, C.-W., Tsai, C.-W., Wang, H.-F., Tsai, H.-C., Chen, H.-Y., Tsai, T.-F., et al. (2004). Identification of a developmentally regulated striatum-enriched zinc-finger gene, Nolz-1, in the mammalian brain. Proc. Natl. Acad. Sci. U S A 101, 2613-2618. doi: 10.1073/pnas.0308645100

Chen, S.-Y., Lu, K.-M., Ko, H.-A., Huang, T.-H., Hao, J.-H., Yan, Y.-T., et al. (2020). Parcellation of the striatal complex into dorsal and ventral districts. Proc. Natl. Acad. Sci. U S A 117, 7418-7429. doi: 10.1073/pnas.1921007117

Ho, N. F., Iglesias, J. E., Sum, M. Y., Kuswanto, C. N., Sitoh, Y. Y., De Souza, J., et al. (2017). Progression from selective to general involvement of hippocampal subfields in schizophrenia. Mol. Psychiatry 22, 142-152. doi: 10.1038/mp. 2016.4

Hoischen, A., Krumm, N., and Eichler, E. E. (2014). Prioritization of neurodevelopmental disease genes by discovery of new mutations. Nat. Neurosci. 17, 764-772. doi: 10.1038/nn.3703

Kai, N., Iwase, K., Imai, K., Nakahira, E., Soma, M., Ohtsuka, S., et al. (2006). Altered gene expression in the subdivisions of the amygdala of Fyn-deficient mice as revealed by laser capture microdissection and mKIAA cDNA array analysis. Brain Res. 1073-1074, 60-70. doi: 10.1016/j.brainres.2005.12.042

Ko, S.-A., Chen, S.-Y., Chen, H.-Y., and Liu, F.-C. (2013). Cell type-selective expression of the zinc finger-containing gene Nolz-1/Zfp503 in the developing mouse striatum. Neurosci. Lett. 548, 44-49. doi: 10.1016/j.neulet.2013.05.020

Lein, E. S., Hawrylycz, M. J., Ao, N., Ayres, M., Bensinger, A., Bernard, A., et al. (2007). Genome-wide atlas of gene expression in the adult mouse brain. Nature 445, 168-176. doi: 10.1038/nature05453

Lencz, T., Knowles, E., Davies, G., Guha, S., Liewald, D. C., Starr, J. M., et al. (2014). Molecular genetic evidence for overlap between general cognitive ability and risk for schizophrenia: a report from the Cognitive Genomics consorTium (COGENT). Mol. Psychiatry 19, 168-174. doi: 10.1038/mp.2013.166

Li, J., Cai, T., Jiang, Y., Chen, H., He, X., Chen, C., et al. (2016). Genes with de novo mutations are shared by four neuropsychiatric disorders discovered from NPdenovo database. Mol. Psychiatry 21, 290-297. doi: 10.1038/mp.2015.40

Liao, W.-L., Tsai, H.-C., Wang, H.-F., Chang, J., Lu, K.-M., Wu, H.-L., et al. (2008). Modular patterning of structure and function of the striatum by retinoid receptor signaling. Proc. Natl. Acad. Sci. U S A 105, 6765-6770. doi: 10.1073/pnas.0802109105

Lobo, M. K., Karsten, S. L., Gray, M., Geschwind, D. H., and Yang, X. W. (2006). FACS-array profiling of striatal projection neuron subtypes in juvenile and adult mouse brains. Nat. Neurosci. 9, 443-452. doi: 10.1038/nn1654

Makarova, K. S., Aravind, L., and Koonin, E. V. (2002). SWIM, a novel Zn-chelating domain present in bacteria, archaea and eukaryotes. Trends Biochem. Sci. 27, 384-386. doi: 10.1016/s0968-0004(02)02140-0

Marin, O., Anderson, S. A., and Rubenstein, J. L. (2000). Origin and molecular specification of striatal interneurons. J. Neurosci. 20, 6063-6076. doi: 10.1523/JNEUROSCI.20-16-06063.2000
Fellowship grants MOST107-2321-B-010-010-MY3 (H-YK), MOST109-2811-B-010-538 (S-YC), MOST104-2811-B-010-026 (K-ML), and the Featured Areas Research Center Program within the framework of the Higher Education Sprout Project by the Ministry of Education in Taiwan (F-CL).

\section{ACKNOWLEDGMENTS}

We thank Drs. H. Koga and T. Nagase for providing the Zswim6 cDNA clone (mKIAA1577) and W. L. Fong and H.-L. Wu for their help with histology.

Mayer, C., Hafemeister, C., Bandler, R. C., Machold, R., Batista Brito, R., Jaglin, X., et al. (2018). Developmental diversification of cortical inhibitory interneurons. Nature 555, 457-462. doi: 10.1038/nature25999

McCutcheon, R. A., Abi-Dargham, A., and Howes, O. D. (2019). Schizophrenia, dopamine and the striatum: from biology to symptoms. Trends Neurosci. 42 , 205-220. doi: 10.1016/j.tins.2018.12.004

Namboodiri, V. M., Rodriguez-Romaguera, J., and Stuber, G. D. (2016). The habenula. Curr. Biol. 26, R873-R877. doi: 10.1016/j.cub.2016.08.051

Olsson, M., Campbell, K., Wictorin, K., and Björklund, A. (1995). Projection neurons in fetal striatal transplants are predominantly derived from the lateral ganglionic eminence. Neuroscience 69, 1169-1182. doi: 10.1016/0306-4522(95) 00325-d

Palmer, E. E., Kumar, R., Gordon, C. T., Shaw, M., Hubert, L., Carroll, R., et al. (2017). A recurrent de novo nonsense variant in Zswim6 results in severe intellectual disability without frontonasal or limb malformations. Am. J. Hum. Genet. 101, 995-1005. doi: 10.1016/j.ajhg.2017.10.009

Pers, T. H., Timshel, P., Ripke, S., Lent, S., Sullivan, P. F., O’Donovan, M. C., et al. (2016). Comprehensive analysis of schizophrenia-associated loci highlights ion channel pathways and biologically plausible candidate causal genes. Hum. Mol. Genet. 25, 1247-1254. doi: 10.1093/hmg/ddw007

Ripke, S., O’Dushlaine, C., Chambert, K., Moran, J. L., Kähler, A. K., Akterin, S., et al. (2013). Genome-wide association analysis identifies 13 new risk loci for schizophrenia. Nat. Genet. 45, 1150-1159. doi: 10.1038/ng.2742

Roeske, M. J., Konradi, C., Heckers, S., and Lewis, A. S. (2020). Hippocampal volume and hippocampal neuron density, number and size in schizophrenia: a systematic review and meta-analysis of postmortem studies. Mol. Psychiatry doi: 10.1038/s41380-020-0853-y [Epub ahead of print].

Rubenstein, J. L. R., and Campbell, K. (2013). "Chapter 24-Neurogenesis in the basal ganglia," in Patterning and Cell Type Specification in the Developing CNS and PNS, eds J. L. R. Rubenstein and P. Rakic (Oxford: Academic Press), $455-473$.

Samaco, R. C., Mandel-Brehm, C., McGraw, C. M., Shaw, C. A., McGill, B. E., and Zoghbi, H. Y. (2012). Crh and Oprm1 mediate anxiety-related behavior and social approach in a mouse model of MECP2 duplication syndrome. Nat. Genet. 44, 206-211. doi: 10.1038/ng.1066

Sandyk, R. (1991). Relevance of the habenular complex to neuropsychiatry: a review and hypothesis. Int. J. Neurosci. 61, 189-219. doi: 10.3109/002074591089 90738

Schizophrenia Working Group of the Psychiatric Genomics Consortium. (2014). Biological insights from 108 schizophrenia-associated genetic loci. Nature 511, 421-427. doi: 10.1038/nature13595

Smith, J. D., Hing, A. V., Clarke, C. M., Johnson, N. M., Perez, F. A., Park, S. S., et al. (2014). Exome sequencing identifies a recurrent de novo Zswim6 mutation associated with acromelic frontonasal dysostosis. Am. J. Hum. Genet. 95, 235-240. doi: 10.1016/j.ajhg.2014.07.008

Steiner, H., Blum, M., Kitai, S. T., and Fedi, P. (1999). Differential expression of ErbB3 and ErbB4 neuregulin receptors in dopamine neurons and forebrain areas of the adult rat. Exp. Neurol. 159, 494-503. doi: 10.1006/exnr. 1999.7163

Stenman, J., Toresson, H., and Campbell, K. (2003). Identification of two distinct progenitor populations in the lateral ganglionic eminence: implications for striatal and olfactory bulb neurogenesis. J. Neurosci. 23, 167-174. doi: 10.1523/JNEUROSCI.23-01-00167.2003 
Takata, A. (2019). Estimating contribution of rare non-coding variants to neuropsychiatric disorders. Psychiatry Clin. Neurosci. 73, 2-10. doi: $10.1111 /$ pcn. 12774

Tao, G., Li, Z., Wen, Y., Song, X., Wei, S., Du, H., et al. (2019). Transcription factors $S p 8$ and $S p 9$ regulate medial ganglionic eminence-derived cortical interneuron migration. Front. Mol. Neurosci. 12:75. doi: 10.3389/fnmol.2019.00075

Tischfield, D. J., Saraswat, D. K., Furash, A., Fowler, S. C., Fuccillo, M. V., and Anderson, S. A. (2017). Loss of the neurodevelopmental gene Zswim6 alters striatal morphology and motor regulation. Neurobiol. Dis. 103, 174-183. doi: 10.1016/j.nbd.2017.04.13

Twigg, S. R., Ousager, L. B., Miller, K. A., Zhou, Y., Elalaoui, S. C., Sefiani, A., et al. (2016). Acromelic frontonasal dysostosis and Zswim6 mutation: phenotypic spectrum and mosaicism. Clin. Genet. 90, 270-275. doi: 10.1111/cge.12721

Vissers, L. E., Gilissen, C., and Veltman, J. A. (2016). Genetic studies in intellectual disability and related disorders. Nat. Rev. Genet. 17, 9-18. doi: 10.1038/nrg3999
Zhang, L., Wang, H., Luan, S., Yang, S., Wang, Z., Wang, J., et al. (2017). Altered volume and functional connectivity of the habenula in schizophrenia. Front. Hum. Neurosci. 11:636. doi: 10.3389/fnhum.2017.00636

Conflict of Interest: The authors declare that the research was conducted in the absence of any commercial or financial relationships that could be construed as a potential conflict of interest.

Copyright (C) 2021 Chang, Kuo, Chen, Lin, Lu, Saito and Liu. This is an open-access article distributed under the terms of the Creative Commons Attribution License (CC BY). The use, distribution or reproduction in other forums is permitted, provided the original author(s) and the copyright owner(s) are credited and that the original publication in this journal is cited, in accordance with accepted academic practice. No use, distribution or reproduction is permitted which does not comply with these terms. 